\title{
Quantum friction between oscillating crystal slabs: Graphene monolayers on dielectric substrates
}

\author{
Vito Despoja, ${ }^{1,2,3}$ Pedro M. Echenique, ${ }^{1,2}$ and Marijan Šunjić1,4 \\ ${ }^{1}$ Donostia International Physics Center (DIPC), P. Manuel de Lardizabal, 20018 San Sebastian, Basque Country, Spain \\ ${ }^{2}$ Departamento de Fisica de Materiales and Centro Mixto CSIC-UPV/EHU, Facultad de Ciencias Quimicas, \\ Universidad del Pais Vasco UPV/EHU, Apto. 1072, 20080 San Sebastian, Basque Country, Spain \\ ${ }^{3}$ Institute of Physics, Bijenička 46, HR-10000 Zagreb, Croatia \\ ${ }^{4}$ Department of Physics, Faculty of Science, University of Zagreb, Bijenička 32, HR-10000 Zagreb, Croatia
}

(Received 7 March 2018; revised manuscript received 7 August 2018; published 5 September 2018)

\begin{abstract}
We present a theoretical description of energy transfer processes between two noncontact quasi-twodimensional crystals separated by distance $a$, oscillating with frequency $\omega_{0}$ and amplitude $\rho_{0}$, and compare it with the case of two quasi-two-dimensional crystals in uniform parallel motion. We apply the theory to calculate van der Waals energy and dissipated energy in two oscillating slabs where each slab consists of a graphene monolayer deposited on $\mathrm{SiO}_{2}$ substrate. The graphene dielectric response is determined from first principles, and $\mathrm{SiO}_{2}$ surface response is described using empirical local dielectric function. We studied the modification of $\mathrm{vdW}$ attraction as a function of the driving frequency and graphene doping. We propose the idea of controlling the binding energy between two slabs by tuning the graphene dopings $E_{F i}$ and driving frequency $\omega_{0}$. We found simple $\rho_{0}^{2}$ dependence of $\mathrm{vdW}$ and dissipated energy. As the Dirac plasmons of frequency $\omega_{p}$ are the dominant channels through which the energy between slabs can be transferred, the dissipated power in equally doped $E_{F 1}=E_{F 2} \neq 0$ graphenes shows strong $\omega_{0}=2 \omega_{p}$ peak. This peak is substantially reduced when graphenes are deposited on the $\mathrm{SiO}_{2}$ substrate. If only one graphene is pristine $\left(E_{F i}=0\right)$ the $2 \omega_{p}$ peak disappears. For larger separations $a$ the phononic losses also become important and the doping causes shifts, appearance, and disappearance of many peaks originating from resonant coupling between hybridized electronic/phononic excitations in graphene/substrate slabs.
\end{abstract}

DOI: 10.1103/PhysRevB.98.125405

\section{INTRODUCTION}

Detailed understanding of noncontact friction and energy transfer processes in nanostructures is of great importance, both from the conceptual and practical viewpoints. Existing theoretical studies, starting with the seminal paper by Pendry [1], mostly consist of calculations of friction coefficients, i.e., friction force between two parallel dielectric plates (e.g., supported graphenes) in uniform relative motion which is experimentally not easily measured (e.g., current drag in one graphene caused by current flow in another one) [2-8].

While the experiments with two slabs in parallel relative motion with constant velocity are difficult to perform, we suggest here that for the same systems experiments with slabs in relative oscillatory motion with fixed or variable frequency might be easier to perform and could lead to new and interesting observations. Recently a similar approach has been realized experimentally [9-12]. In these experiments the system (usually an AFM tip above the surface) oscillates at some characteristic frequency. These oscillations are then, because of various dissipation mechanisms (which includes quantum friction), damped. Our model is based on a slightly different concept; one of the slabs, e.g., the AFM tip, is driven with variable frequency. This means that the friction can be deduced from the energy dissipated in one oscillating cycle. In this paper we provide a general theoretical description of such processes, expecting that this method might become a useful tool to study dynamical properties of low-dimensional systems [13].
The main objective of this paper is therefore a theoretical description of these phenomena in systems consisting of two nontouching polarizable media, specifically conservative (van der Waals or Casimir) and dissipative forces (quantum friction) between two quasi-two-dimensional (q2D crystals) in relative parallel and oscillatory motion. While the case of slabs in parallel uniform motion has been extensively studied [1,5,14-18], here we develop an analogous theory describing interaction of atomically thick slabs (q2D crystals) in oscillatory motion.

It should be emphasized that here we study the vdW energy for smaller slab separations $(a<100 \mathrm{~nm})$ where the retardation effects are not so important and the whole formulation is performed in the electrostatic limit. Therefore the vdW energies are more robust and not comparable with fine Casimir effects. In order to study the Casimir forces the formulation should be performed in the retardation limit. Moreover, the Casimir effects are very sensitive to the characteristics of low frequency $(\omega)$ and low wave vector $(Q)$ plasmon/phonon modes where intensity depends on the damping constant used in the calculation [8,19-23]. Damping constant depends on various relaxation mechanisms, such as electron-phonon or electron-impurity scattering processes which are also very sensitive on temperature $[24,25]$. Therefore the study of Casimir effects is outside the scope of this study and should be done as a separated study which carefully includes all these effects.

In Sec. II the expressions for van der Waals and dissipative energies and forces are derived for such a q2D system in a very 
general case, for variable slab temperatures and dynamical properties characterized by their surface response functions $D_{1}$ and $D_{2}$, and for variable oscillating frequencies and amplitudes. We assume 2D translational invariance and neglect retardation for the slab distances in consideration. For the sake of clarity and comparison, in Appendix we derive analogous results for the case of parallel uniform motion, recovering but also generalizing some earlier results $[26,27]$.

In Sec. III we derive general expressions for surface response functions $D_{i}$ for multilayer slabs, later to be specified for monolayers of a substance like graphene or silicene adsorbed on dielectric substrates. Surface response functions $D_{1}$ and $D_{2}$ will be the key ingredients in the expressions describing dissipative and reactive processes in Sec. II and Sec. III. In Sec. III we also show how to calculate surface response functions $D_{i}$ for a specific case of q2D crystals on a dielectric substrate The expression for the surface excitation propagator of a system of two coupled slabs is also derived.

In Sec. IV we present the models used to describe the q2D crystal and substrate dynamical response. We study the specific case of a graphene monolayer on a dielectric substrate, which is chosen to be ionic crystal $\mathrm{SiO}_{2}$. The substrate is considered as a homogenous semi-infinite ionic crystal $\mathrm{SiO}_{2}$ with the appropriate dielectric function in the longwavelength limit. Graphene monolayer dynamical response is determined from first principles. Also some computational details are specified.

In Sec. V general expressions of previous sections are applied to the system of two slabs, where each slab represents a graphene $\left(E_{F i}\right) / \mathrm{SiO}_{2}$ system and where graphene doping is characterized by Fermi energy $E_{F}$ relative to the Dirac point. In Sec. V A we demonstrate how the spectra of electronic excitations in one slab and in two coupled slabs depend on graphene doping $E_{F}$.

The form of these coupled excitations is responsible for the behavior of the attractive forces and dissipation. We first discuss in Sec. VB the modification of van der Waals force for oscillating in comparison with the static slabs. Van der Waals energies depend on two factors. They increase with the increased graphene doping but are reduced for the asymmetric doping when excitations in two slabs are off resonance. Dynamical vdW energy shows unusual behavior: It starts as plateau and then decreases. This is because the fast Dirac plasmon of frequency $\omega_{p}$ in one slab, for low driving frequencies $\omega_{0}<\omega_{p}$, still perfectly follows Doppler shifted charge density fluctuations in another slab. For larger driving frequencies this is not the case and $\mathrm{vdW}$ energies decrease. Finally, for small or zero doping the $\pi \rightarrow \pi^{*}$ and $\pi \rightarrow \sigma$ excitations cause linear weakening of the dynamical $\mathrm{vdW}$ energy.

In Sec. V C we explore how the details of realistic graphene band structure influence the dissipated power, i.e., what are the limitations of Dirac-cone approximation which treats the $\pi$ electron bands as entirely conical. In Sec. V D we calculate and discuss how dissipated power depends on various parameters: driving amplitude $\rho_{0}$ and frequency $\omega_{0}$, on the separations between slabs $a$ and on the substrate. We find simple $\rho_{0}^{2}$ dependence, while the $\omega_{0}$ dependence is determined by the intensity of resonant coupling between hybridized Dirac plasmons and substrate transverse optical (TO) phonons. We

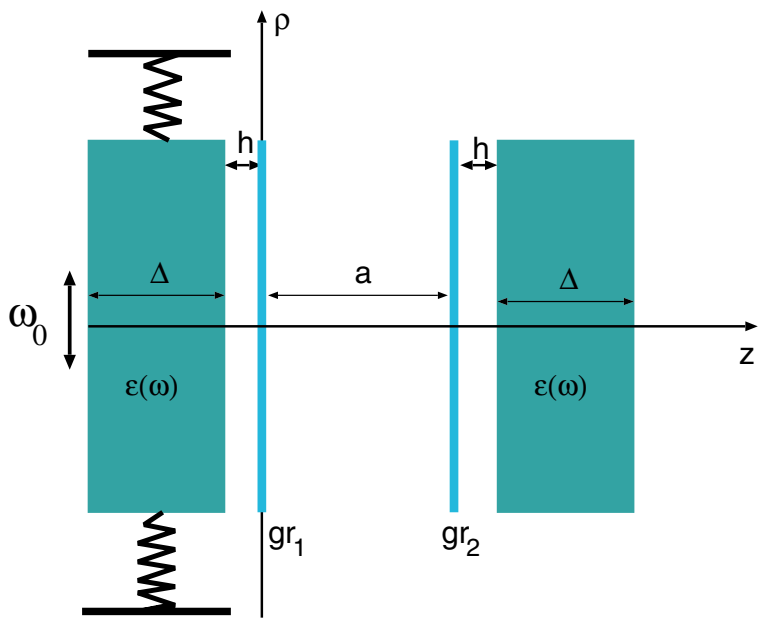

FIG. 1. Geometry of the system.

also explain why the substrate substantially reduces dissipated power peak. For larger separations $a$ additional peaks appear in dissipated power originating from the excitations of hybridized substrate phonons.

In Sec. VE we explore how the dissipated power depends on graphene dopings. We show that if one graphene is pristine $\left(E_{F}=0\right)$ it causes the disappearance of strong $2 \omega_{p}$ peak in the dissipated power. Moreover, for larger separations the doping causes shifts, appearance and disappearance of many peaks originating from resonant coupling between hybridized substrate phonons and Dirac plasmons. In Sec. VI we present the conclusions.

\section{GENERAL THEORY: OSCILLATING SLABS}

\section{A. Van der Waals energy and force}

In Appendix A 1 we have derived van der Waals energy and force between two slabs in uniform relative motion in some detail because it will help us to treat a similar problem of two oscillating slabs. Similar formulas have been derived by considering the electromagnetic field fluctuations in two slabs in relative motion in the retarded limit $[5,28]$. These formulas in the nonretarded limit reduce to our formulas (A16) or (A21).

We shall later assume that the slabs consist of graphene monolayers with variable doping, deposited on dielectric slabs of thickness $\Delta$ described by local dielectric functions $\epsilon(\omega)$, as shown in Fig. 1. The left slab mechanically oscillates with frequency $\omega_{0}$ and amplitude $\rho_{0}$ relative to the right slab. Again we calculate the diagram in Fig. 11 as in Sec. A 1, but now the slab parallel coordinates change in time as

$$
\boldsymbol{\rho}-\boldsymbol{\rho}_{1} \rightarrow \boldsymbol{\rho}-\boldsymbol{\rho}_{1}-\boldsymbol{\rho}_{0}\left(\sin \omega_{0} t-\sin \omega_{0} t_{1}\right)
$$

so that instead of (A3) we have

$$
\begin{aligned}
E_{c}= & \int_{-\infty}^{\infty} d t_{1} \int \frac{d \mathbf{Q}}{(2 \pi)^{2}} e^{-i \mathbf{Q} \rho_{0}\left(\sin \omega_{0} t-\sin \omega_{0} t_{1}\right)} \\
& \times \int_{-\infty}^{\infty} d z d z_{1} d z_{2} d z_{3} S_{1}\left(\mathbf{Q}, z, z_{1}, t-t_{1}\right) V\left(\mathbf{Q}, z, z_{3}\right) \\
& \times D_{2}\left(\mathbf{Q}, z_{3}, z_{2}, t-t_{1}\right) V\left(\mathbf{Q}, z_{2}, z_{1}\right),
\end{aligned}
$$


where $\boldsymbol{\rho}=(x, y)$ is the position vector and $\mathbf{Q}=\left(Q_{x}, Q_{y}\right)$ is the wave vector in the plane parallel to crystal slab.

If we use

$$
e^{i z \sin \phi}=\sum_{m=-\infty}^{\infty} J_{m}(z) e^{i m \phi}
$$

where $J_{m}$ are Bessel functions, after Fourier transformation in $\omega$ space, using expressions (A5)-(A7), (A9), and integration over $z$ coordinates we obtain

$$
\begin{aligned}
E_{c}= & \hbar \int \frac{d \mathbf{Q}}{(2 \pi)^{2}} e^{-2 Q a} \sum_{m, m^{\prime}=-\infty}^{\infty} J_{m}\left(\mathbf{Q} \boldsymbol{\rho}_{0}\right) J_{m^{\prime}}\left(\mathbf{Q} \boldsymbol{\rho}_{0}\right) \\
& \times \int_{-\infty}^{\infty} \frac{d \omega}{2 \pi}\left[2 n_{1}(\omega)+1\right] e^{i\left(m-m^{\prime}\right) \omega_{0} t} \\
& \times I m D_{1}(\mathbf{Q}, \omega) \operatorname{Re} D_{2}\left(\mathbf{Q}, \omega+m \omega_{0}\right)
\end{aligned}
$$

Here we have also used the fact that $\operatorname{Im}_{2}(\mathbf{Q}, \omega)$ is an antisymmetric function of $\omega$ and does not contribute to integration. We see that the energy oscillates in time with frequencies $\left(m-m^{\prime}\right) \omega_{0}$. If we assume to measure energies on a time scale $\Delta t>T$, where $T=\frac{2 \pi}{\omega_{0}}$ is the maximal duration of one cycle, then we can average over $T$

$$
\frac{1}{T} \int_{0}^{T} d t e^{i\left(m-m^{\prime}\right) \omega_{0} t}=\delta_{m m^{\prime}},
$$

and find the result independent of time:

$$
\begin{aligned}
E_{c}= & \frac{\hbar}{2} \int \frac{d \mathbf{Q}}{(2 \pi)^{2}} e^{-2 Q a} \sum_{m=0}^{\infty}\left(2-\delta_{m 0}\right) J_{m}^{2}\left(\mathbf{Q} \rho_{0}\right) \\
& \times \int_{-\infty}^{\infty} \frac{d \omega}{2 \pi}\left\{\left[2 n_{1}(\omega)+1\right]\right. \\
& \times \operatorname{Im} D_{1}(\mathbf{Q}, \omega) \operatorname{Re} D_{2}\left(\mathbf{Q}, \omega+m \omega_{0}\right) \\
& \left.+\left[2 n_{2}(\omega)+1\right] \operatorname{Im} D_{2}(\mathbf{Q}, \omega) \operatorname{Re} D_{1}\left(\mathbf{Q}, \omega+m \omega_{0}\right)\right\}
\end{aligned}
$$

where the expression in curly brackets is fully analogous to the one in (A11), but now $\omega^{\prime} \rightarrow \omega_{m}=\omega+m \omega_{0}$. In the next higher order process the field which is scattered is scattered once again which, after applying the convolution theorem, collects the phase factor $e^{i\left(m+n-m^{\prime}-n^{\prime}\right) \omega_{0} t}$. The approximation which we use here in order to eliminate this oscillatory term is that we put $m=m^{\prime}$ and $n=n^{\prime}$, and similarly in all higher order terms. In this kind of approximation mixed $J_{n} J_{m}$ terms which oscillate faster than $J_{n}^{2}$ disappear. Also, in the inclusion of higher order processes we can follow the same procedure as for the parallel motion in Sec. A 1. After applying this approximation and integration over the coupling constant, we obtain the result analogous to (A16)

$$
\begin{aligned}
E_{c}= & \frac{\hbar}{2} \int \frac{d \mathbf{Q}}{(2 \pi)^{2}} \sum_{m=0}^{\infty}\left(2-\delta_{m 0}\right) J_{m}^{2}\left(\mathbf{Q} \rho_{0}\right) \\
& \times \int_{-\infty}^{\infty} \frac{d \omega}{2 \pi} A\left(\mathbf{Q}, \omega, \omega_{m}\right),
\end{aligned}
$$

where $A$ is given by (A17) and (A18), with $\omega_{m}=\omega+m \omega_{0}$.

Again, the limiting cases can be obtained from Sec. A 1. For $\omega_{0}=0\left(\omega^{\prime}=\omega\right)$ and $\rho_{0}=0$ we find the well known result for van der Waals interaction when the slabs are at rest
$[29,30]:$

$$
\begin{aligned}
E_{c}(a)= & \frac{\hbar}{2} \int \frac{d \mathbf{Q}}{(2 \pi)^{2}} \int_{0}^{\infty} \frac{d \omega}{2 \pi} \operatorname{sgn} \omega \\
& \times \operatorname{Im} \ln \left[1-e^{-2 Q a} D_{1}(\mathbf{Q}, \omega) D_{2}(\mathbf{Q}, \omega)\right] .
\end{aligned}
$$

For finite frequency $\omega_{0}$ and $D_{1}=D_{2}=D$ we find:

$$
\begin{aligned}
E_{c}(a)= & \frac{\hbar}{2} \int \frac{d \mathbf{Q}}{(2 \pi)^{2}} \sum_{m=0}^{\infty}\left(2-\delta_{m 0}\right) J_{m}^{2}\left(\mathbf{Q} \rho_{0}\right) \int_{-\infty}^{\infty} \frac{d \omega}{2 \pi} s g n \omega \\
& \times \operatorname{Im} \ln \left[1-e^{-2 Q a} D(\mathbf{Q}, \omega) D\left(\mathbf{Q}, \omega_{m}\right)\right] .
\end{aligned}
$$

We notice that the frequency integrals are the same as in (A16)-(A20). Also, the attractive van der Waals force between two oscillating slabs is given by

$$
\begin{aligned}
F_{\perp}(a)= & -\frac{d E_{c}(a)}{d a} \\
= & \hbar \int \frac{d \mathbf{Q}}{(2 \pi)^{2}} Q e^{-2 Q a} \sum_{n=0}^{\infty}\left(2-\delta_{m 0}\right) J_{m}^{2}\left(\mathbf{Q} \boldsymbol{\rho}_{0}\right) \\
& \times \int_{-\infty}^{\infty} \frac{d \omega}{2 \pi} B\left(\mathbf{Q}, \omega, \omega_{m}\right),
\end{aligned}
$$

where the function $B$ is given by (A22) and (A23). The same holds for the $\omega_{0} \rightarrow 0$ or $D_{1}=D_{2}=D$ limits when the expressions for $B$ become (A24) or (A25), respectively.

\section{B. Dissipated power}

We can perform the calculation of the dissipated power for two slabs oscillating parallel to each other with amplitude $\boldsymbol{\rho}_{0}$ and frequency $\omega_{0}$ in analogy with the previous treatment of two slabs in uniform relative motion in Sec. A 2. Again, we have to transform the parallel coordinates in the left slabs as in (1). Then (A29), after integration over $t_{1}$ becomes

$$
\begin{aligned}
P_{12}(t)= & -i \hbar \int \frac{d \mathbf{Q}}{(2 \pi)^{2}} \int \frac{d \omega}{2 \pi} \sum_{m, m^{\prime}=-\infty}^{\infty}(-1)^{m+m^{\prime}} \\
& \times e^{i\left(m^{\prime}-m\right) \omega_{0} t}\left(m^{\prime} \omega_{0}-\omega\right) J_{m}\left(\mathbf{Q} \boldsymbol{\rho}_{0}\right) J_{m}^{\prime}\left(\mathbf{Q} \boldsymbol{\rho}_{0}\right) \\
& \times S_{1}\left(\mathbf{Q},|\omega|, z, z_{1}\right) \otimes V\left(\mathbf{Q}, z, z_{3}\right) \\
\otimes & D_{2}\left(\mathbf{Q}, m^{\prime} \omega_{0}-\omega, z_{3}, z_{2}\right) \otimes V\left(\mathbf{Q}, z_{2}, z_{1}\right)
\end{aligned}
$$

We see that the energy absorption rate in the left slab is time dependent and oscillates with frequency $\left(m^{\prime}-m\right) \omega_{0}$. Again, from (2) we see that for time intervals large with respect to the oscillation period $T$ the terms $m \neq m^{\prime}$ do not contribute and the energy absorption rate is

$$
\begin{aligned}
P_{12}= & -i \hbar \int \frac{d \mathbf{Q}}{(2 \pi)^{2}} \int \frac{d \omega}{2 \pi} \sum_{m=-\infty}^{\infty}\left(m \omega_{0}-\omega\right) J_{m}^{2}\left(\mathbf{Q} \boldsymbol{\rho}_{0}\right) \\
& \times S_{1}\left(\mathbf{Q},|\omega|, z, z_{1}\right) \otimes V\left(\mathbf{Q}, z, z_{3}\right) \\
\otimes & D_{2}\left(\mathbf{Q}, m \omega_{0}-\omega, z_{3}, z_{2}\right) \otimes V\left(\mathbf{Q}, z_{2}, z_{1}\right) .
\end{aligned}
$$

If we now use (A5), the definitions (A6) and (A7) of the surface excitation propagator and surface correlation function, respectively, and the connection (A9) between the surface correlation function and the imaginary part of surface excitation 
propagator, equation (6) can be written as

$$
\begin{aligned}
P_{12}= & -\frac{\hbar}{\pi} \sum_{m=-\infty}^{\infty} \int \frac{d \mathbf{Q}}{(2 \pi)^{2}} e^{-2 Q a} J_{m}^{2}\left(\mathbf{Q} \rho_{0}\right) \int \frac{d \omega}{2 \pi} \omega_{m} \\
& \times\left[2 n_{1}(\omega)+1\right] \operatorname{Im} D_{1}(\mathbf{Q}, \omega) \operatorname{Im} D_{2}\left(\mathbf{Q}, \omega_{m}\right) .
\end{aligned}
$$

Evaluating (7) we have used the fact that the real part of the function under summation and integration is odd and the imaginary part is an even function of $n$ and $\omega . P_{12}$ is the energy absorption rate in the left slab in the reference frame of the right slab or in the laboratory reference of the frame. Now we have to repeat the discussion in Sec. A 2 and subtract the same energy absorption rate but calculated in the reference frame of the left (oscillating) slab. The same arguments, leading to (A37), will give this energy to be

$$
\begin{aligned}
P_{12}^{\prime}= & \hbar \sum_{n=-\infty}^{\infty} \int \frac{d \mathbf{Q}}{(2 \pi)^{2}} e^{-2 Q a} J_{n}^{2}\left(\mathbf{Q} \boldsymbol{\rho}_{0}\right) \int \frac{d \omega}{2 \pi} \omega \\
& \times\left[2 n_{1}(\omega)+1\right] \operatorname{Im} D_{1}(\mathbf{Q}, \omega) \operatorname{Im} D_{2}\left(\mathbf{Q}, \omega_{n}\right)
\end{aligned}
$$

Expression (8) represents the energy which fluctuates between left and right slabs but is not spent to heating. The dissipated (heating) power can be calculated by extracting from $P_{12}$ this fluctuating component, i.e.,

$$
\begin{aligned}
P_{1}= & P_{12}-P_{12}^{\prime}=2 \hbar \sum_{m=1}^{\infty} m \omega_{0} \int \frac{d \mathbf{Q}}{(2 \pi)^{2}} e^{-2 Q a} J_{m}^{2}\left(\mathbf{Q} \boldsymbol{\rho}_{0}\right) \\
& \times \int \frac{d \omega}{2 \pi}\left[2 n_{1}(\omega)+1\right] \operatorname{Im} D_{1}(\mathbf{Q}, \omega) \operatorname{Im} D_{2}\left(\mathbf{Q}, \omega_{m}\right) .
\end{aligned}
$$

Analogous calculation would give the energy dissipated in the process where the charge fluctuation in the right slab induces fluctuations in the left slab. We have to exchange 1 and 2 in (9) and replace $m \rightarrow-m$. Repeating the steps in (A40) the final result becomes:

$$
\begin{aligned}
P= & P_{1}+P_{2} \\
= & 4 \hbar \sum_{m=1}^{\infty} m \omega_{0} \int \frac{d \mathbf{Q}}{(2 \pi)^{2}} e^{-2 Q a} J_{m}^{2}\left(\mathbf{Q} \boldsymbol{\rho}_{0}\right) \int_{-\infty}^{\infty} \frac{d \omega}{2 \pi} \\
& \times\left[n_{1}(\omega)-n_{2}\left(\omega_{m}\right)\right] \operatorname{Im} D_{1}(\mathbf{Q}, \omega) \operatorname{Im} D_{2}\left(\mathbf{Q}, \omega_{m}\right) .
\end{aligned}
$$

This expression is analogous to (A40). For $T=02 n(\omega)+$ $1 \rightarrow$ sgnw and (10) can be written as

$$
\begin{aligned}
P= & 4 \hbar \sum_{m=1}^{\infty} m \omega_{0} \int \frac{d \mathbf{Q}}{(2 \pi)^{2}} e^{-2 Q a} J_{m}^{2}\left(\mathbf{Q} \rho_{0}\right) \int_{0}^{m \omega_{0}} \frac{d \omega}{2 \pi} \\
& \times \operatorname{Im} D_{1}(\mathbf{Q}, \omega) I m D_{2}\left(\mathbf{Q}, m \omega_{0}-\omega\right) .
\end{aligned}
$$

Adding higher order terms [(A12),(A13)] we obtain the energy dissipated per unit time:

$$
\begin{aligned}
P= & 2 \hbar \sum_{m=1}^{\infty} m \omega_{0} \int \frac{d \mathbf{Q}}{(2 \pi)^{2}} e^{-2 Q a} J_{m}^{2}\left(\mathbf{Q} \boldsymbol{\rho}_{0}\right) \\
& \times \int_{-\infty}^{\infty} \frac{d \omega}{2 \pi} C\left(\mathbf{Q}, \omega, \omega_{m}\right)
\end{aligned}
$$

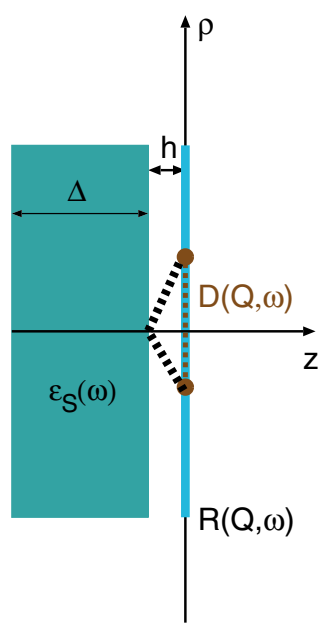

FIG. 2. Simplified model where the $\mathrm{SiO}_{2}$ substrate is shown as a homogenous dielectric slab described by the local dielectric function $\epsilon_{S}(\omega)$ and graphene is described by $2 \mathrm{D}$ response function $R(\mathbf{Q}, \omega) . D(\mathbf{Q}, \omega)$ is the surface excitation propagator of the substrate/graphene composite.

where $C$ is given by (A42). Limiting cases are also obtained from (12). For $\omega_{0}=0$ and/or for $\rho_{0}=0$ obviously $P=0$.

\section{DERIVATION OF THE SLAB SURFACE EXCITATION PROPAGATORS $D_{1,2}(Q, \omega)$}

The main quantities which appear in the formula for van der Waals interaction $E_{c}$ or dissipated power $P$ are the surface excitation propagators $D_{1}(\mathbf{Q}, \omega)$ and $D_{2}(\mathbf{Q}, \omega)$ of the left (first) and right (second) slab, respectively. The derivation of $D_{1}$ and $D_{2}$ is analogous for both slabs, so here we shall derive just one surface excitation propagator $D$. The structure of the monolayer-substrate composite (e.g., graphene on $\mathrm{SiO}_{2}$ ) is shown in Fig. 2. The slab consists of the graphene monolayer adsorbed at distance $h$ (e.g., $h=0.4 \mathrm{~nm}$ ) above the substrate of macroscopic thickness $\Delta$. Although we chose this value for $h$, the results are insensitive to small variation about this value, so we could freely put $h=0$. The dielectric, e.g., the $\mathrm{SiO}_{2}$ slab is placed in the region $-\Delta-h \leqslant z \leqslant-h$ and the graphene layer occupies $z=0$ plane. The same model system is used in Refs. [31,32] where the authors explore plasmon-phonon hybridization, stopping power, and wake effect produced by the proton moving parallel to the composite. The unit cell for such huge nanostructure would consist of hundreds of atoms, so it is impossible to perform full ab initio ground state and structure optimization calculation. Moreover, an $a b$ initio calculation of the response function would be even more demanding so we need an approximation for the response function calculation. The easiest (and probably the best) approximation is to treat the $\mathrm{SiO}_{2}$ slab as a homogeneous dielectric described by some local dielectric function $\epsilon_{S}(\omega)$ and to consider graphene as a purely $2 \mathrm{D}$ system described by the response function $R(\mathbf{Q}, \omega)$, as sketched in Fig. 2 . 
In order to derive the surface excitation propagator $D(\mathbf{Q}, \omega)$ we start from its definition:

$$
\begin{aligned}
D(\mathbf{Q}, \omega) & =v_{Q} \int_{-\infty}^{0} d z d z^{\prime} e^{-Q\left(z+z^{\prime}\right)} R\left(\mathbf{Q}, \omega, z, z^{\prime}\right) \\
& =\frac{1}{v_{Q}}\left\{W\left(\mathbf{Q}, \omega, z=0, z^{\prime}=0\right)-v_{Q}\right\} ; i=1,2,
\end{aligned}
$$

where $v_{Q}=2 \pi / Q, Q=\sqrt{Q_{x}^{2}+Q_{y}^{2}}$ and $R\left(\mathbf{Q}, \omega, z, z^{\prime}\right)$ represents the nonlocal dielectric function of graphene/dielectric composite which we assume occupies the region $z, z^{\prime} \leqslant 0$. It can be noticed that surface excitation propagator (13) is actually proportional to the induced Coulomb interaction $W^{\text {ind }}=W-v_{Q}$ at the $z=z^{\prime}=0$ surface.

It is well known $[24,25,33,34]$ that physical properties of a graphene monolayer in the low $(Q, \omega)$ region can be described to a very good approximation assuming the monolayer to be strictly two dimensional, so that the nonlocal independent electron response function can be written as

$$
R^{0}\left(\mathbf{Q}, \omega, z, z^{\prime}\right)=R^{0}(\mathbf{Q}, \omega) \delta(z) \delta\left(z^{\prime}\right),
$$

where we assume that the graphene lies in the $z=0$ plane and the response function $R^{0}(\mathbf{Q}, \omega)$ can be derived from first principles, as described in Sec. IV. Dynamically screened response function $R(\mathbf{Q}, \omega)$ in RPA is given as a series of terms

$$
R(\mathbf{Q}, \omega)=R^{0}+R^{0} v_{Q} R^{0}+\ldots=\frac{R^{0}(\mathbf{Q}, \omega)}{1-v_{Q} R^{0}(\mathbf{Q}, \omega)} .
$$

If we assume for the moment that there is no dielectric in the system [e.g., $\epsilon_{S}(\omega)=1$ ] then the screened Coulomb interaction is simply given by

$$
W\left(\mathbf{Q}, \omega, z=0, z^{\prime}=0\right)=v_{Q}+v_{Q} R(\mathbf{Q}, \omega) v_{Q} .
$$

Using the definition (13) the surface excitation propagator becomes

$$
D(\mathbf{Q}, \omega)=v_{Q} R(\mathbf{Q}, \omega) .
$$

When the dielectric slab is introduced, the external charges and charge density fluctuations in the graphene layer do not interact via the bare Coulomb interaction $v_{Q}$ but via the Coulomb interaction modified by the presence of the dielectric slab [35]

$$
v_{Q} \rightarrow \tilde{v}_{Q}(\omega)=v_{Q}\left[1+D_{S}(\mathbf{Q}, \omega)\right],
$$

where the substrate surface excitation propagator is

$$
D_{S}(\mathbf{Q}, \omega)=D_{S}(\omega) \frac{1-e^{-2 Q \Delta}}{1-D_{S}^{2}(\omega) e^{-2 Q \Delta}} e^{-2 Q h}
$$

and

$$
D_{S}(\omega)=\frac{1-\epsilon_{S}(\omega)}{1+\epsilon_{S}(\omega)}
$$

represents the surface excitation propagator of a semi-infinite $(\Delta \rightarrow \infty, h=0)$ dielectric. This causes that the screened Coulomb interaction (16) becomes the function of $\tilde{v}_{Q}(\omega)$

$$
W \rightarrow \tilde{W}=\tilde{v}_{Q}(\omega)+\tilde{v}_{Q}(\omega) \tilde{R}(\mathbf{Q}, \omega) \tilde{v}_{Q}(\omega),
$$

where, because charge density fluctuations inside graphene also interact via $\tilde{v}_{Q}(\omega)$, the screened response function is modified as

$$
\tilde{R}(\mathbf{Q}, \omega)=\frac{R^{0}(\mathbf{Q}, \omega)}{1-\tilde{v}_{Q}(\omega) R^{0}(\mathbf{Q}, \omega)} .
$$

Finally, after inserting (21) into (13) we obtain the surface excitation propagator in the presence of the dielectric

$$
D(\mathbf{Q}, \omega)=\frac{1}{v_{Q}}\left\{\tilde{v}_{Q}(\omega) \tilde{R}_{i}(\mathbf{Q}, \omega) \tilde{v}_{Q}(\omega)+\tilde{v}_{Q}(\omega)-v_{Q}\right\},
$$

which can be rewritten in a more transparent form as

$$
\begin{aligned}
& D(\mathbf{Q}, \omega) \\
& \quad=\frac{D_{S}(\mathbf{Q}, \omega)+v_{Q} R(\mathbf{Q}, \omega)+2 v_{Q} R(\mathbf{Q}, \omega) D_{S}(\mathbf{Q}, \omega)}{1-v_{Q} R(\mathbf{Q}, \omega) D_{S}(\mathbf{Q}, \omega)},
\end{aligned}
$$

which corresponds exactly to Eq. (10) of Ref. [3]. The spectrum of coupled excitations in a single slab can be calculated from

$$
S(\mathbf{Q}, \omega)=-\frac{1}{\pi} \operatorname{Im} D(\mathbf{Q}, \omega)
$$

For the coupled slabs described by their surface excitations propagators $D_{1}$ and $D_{2}$, separated by the distance $a$, in a similar way we can derive the propagator $\tilde{D}$ for the coupled system

$$
\tilde{D}(\mathbf{Q}, \omega)=\frac{D_{1}(\mathbf{Q}, \omega)+D_{2}(\mathbf{Q}, \omega)+2 D_{1}(\mathbf{Q}, \omega) D_{2}(\mathbf{Q}, \omega)}{1-e^{-2 Q a} D_{1}(\mathbf{Q}, \omega) D_{2}(\mathbf{Q}, \omega)}
$$

and the excitation spectrum of this system is

$$
\tilde{S}(\mathbf{Q}, \omega)=-\frac{1}{\pi} \operatorname{Im} \tilde{D}(\mathbf{Q}, \omega) .
$$

\section{DESCRIPTION OF SUBSTRATE AND GRAPHENE DYNAMICAL RESPONSE}

The results in Sec. III are quite general and can be applied to a monolayer of any material on any dielectric substrate. Now we shall specify the dielectric substrate to be the homogenous layer of ionic crystal $\mathrm{SiO}_{2}$.

Dielectric properties (or dynamical response) of bulk ionic crystals in the long-wavelength limit can be described in terms of their optical phonons at the $\Gamma$ point. More complex polar crystals such as $\mathrm{SiO}_{2}$ possess a multitude of different optical phonons of different symmetries and polarizations. However, here we suppose that $\mathrm{SiO}_{2}$ possesses two welldefined, nondispersing TO phonon modes at the frequencies $\omega_{T O 1}$ and $\omega_{T O 2}$ with the corresponding damping rates $\gamma_{T O 1}$ and $\gamma_{T O 2}$, giving rise to a dielectric function of the form $[31,32]$

$$
\begin{aligned}
\epsilon_{S}(\omega)= & \epsilon_{\infty}+\left(\epsilon_{i}-\epsilon_{\infty}\right) \frac{\omega_{T O 2}^{2}}{\omega_{T O 2}^{2}-\omega^{2}-i \omega \gamma_{T O 2}} \\
& +\left(\epsilon_{0}-\epsilon_{i}\right) \frac{\omega_{T O 1}^{2}}{\omega_{T O 1}^{2}-\omega^{2}-i \omega \gamma_{T O 1}}
\end{aligned}
$$




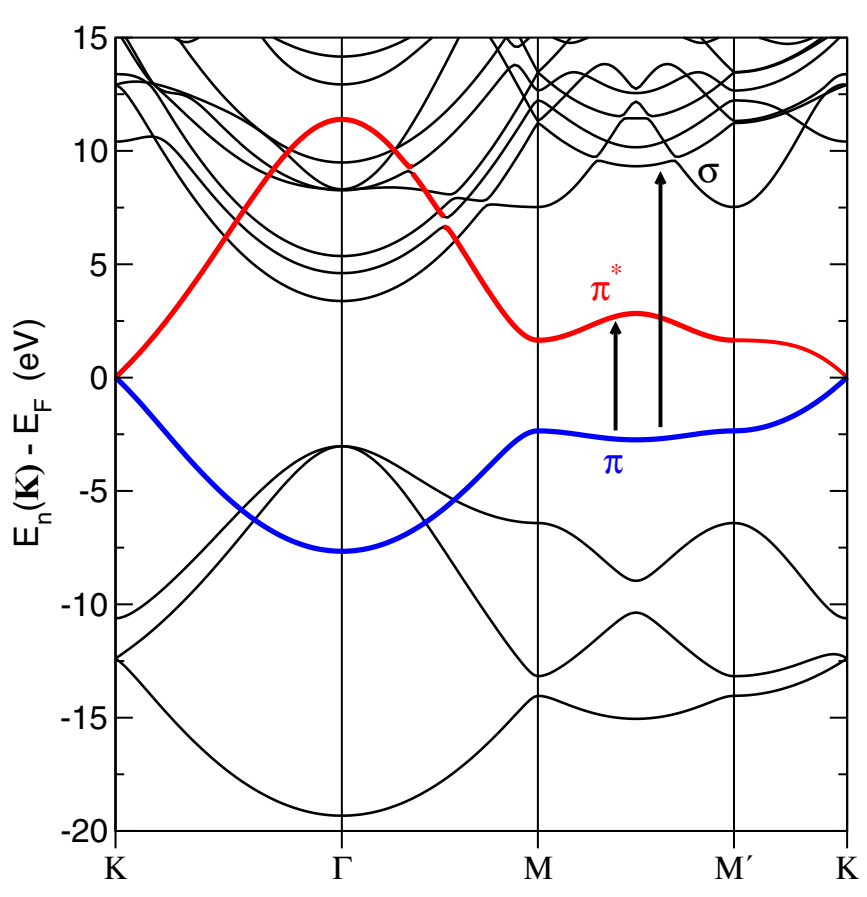

FIG. 3. Pristine graphene band structure. The arrows indicate high energy interband transitions between valence $\pi$ and conducting $\pi^{*}$ and $\sigma$ bands, which could be an important contribution to vdW dissipation energy for larger driving frequencies $\omega_{0}$. (Taken from Ref. [38].)

where $\epsilon_{0}, \epsilon_{i}$, and $\epsilon_{\infty}$ represent the dielectric constant for $\mathrm{SiO}_{2}$ at the zero, intermediate, and very large frequencies. This dielectric function will be inserted in the expression (19) for the substrate surface excitation propagator $D_{S}(\mathbf{Q}, \omega)$. In continuation we shall describe the $a b$ initio approach we use to determine graphene response function response and conductivity tensor, but it should first be mentioned how it relates to various analytical approaches.

There are many analytical results for graphene conductivity or response function in the Dirac cone approximation [36] or even in the extended two band TBA model where $\pi$ and $\pi^{*}$ bands spread throughout the whole Brillouin zone [24,25,37], as shown by blue and red lines in Fig. 3. Such more elegant analytical or semianalitical approaches are more appropriate to study vdW energy or dissipation especially in the low oscillating frequency $\omega_{0}<10 \mathrm{THz}$ limit when low energy hybridised Dirac plasmon/phonon modes and intraband $\pi^{*} \rightarrow$ $\pi^{*}$ transitions become the most important contributions. On the other hand, $a b$ initio methodology, which nowadays for $2 \mathrm{D}$ crystals such as graphene has become computationally relatively easy, allows very accurate calculations of response function and conductivity tensor which excellently agree with the Dirac cone or two band model analytical approaches. However, at the same time they give more accurate results for larger frequencies $\omega$ and wave vectors $Q$, as demonstrated in Ref. [33]. This is especially important in pristine graphene and for larger oscillation frequencies $\omega_{0}$ when the dominant contribution to vdW energy or dissipation comes from high energy $\pi \rightarrow \pi^{*}$ and $\pi \rightarrow \sigma$ electron-hole excitations (and corresponding $\pi$ and $\pi+\sigma$ plasmons) beyond the Dirac cone approach, as sketched in Fig. 3. Therefore in order to be consistent with analytical approaches but at the same time more accurate in the high excitation energy limit we decided to use a more sophisticated $a b$ initio approach.

The graphene response function $R(\mathbf{Q}, \omega)$ is given by (22) in terms of the noninteracting response function

$$
R^{0}(\mathbf{Q}, \omega)=L R_{\mathbf{G}=0 \mathbf{G}^{\prime}=0}^{0}(\mathbf{Q}, \omega),
$$

where the 3D Fourier transform of independent electron response function is given by [39]

$$
\begin{aligned}
& R_{\mathbf{G G}^{\prime}}^{0}(\mathbf{Q}, \omega) \\
& =\frac{2}{\Omega} \sum_{\mathbf{K} \in S . B . Z .} \sum_{n, m} \frac{f_{n}(\mathbf{K})-f_{m}(\mathbf{K}+\mathbf{Q})}{\hbar \omega+i \eta+E_{n}(\mathbf{K})-E_{m}(\mathbf{K}+\mathbf{Q})} \\
& \times \rho_{n \mathbf{K}, m \mathbf{K}+\mathbf{Q}}(\mathbf{G}) \rho_{n \mathbf{K}, m \mathbf{K}+\mathbf{Q}}^{*}\left(\mathbf{G}^{\prime}\right),
\end{aligned}
$$

where $f_{n \mathbf{K}}=\left[e^{\left(E_{n \mathbf{K}}-E_{F}\right) / k T}+1\right]^{-1}$ is the Fermi-Dirac distribution at temperature $T$. The charge vertices in (30) have the form

$$
\rho_{n \mathbf{K}, m \mathbf{K}+\mathbf{Q}}(\mathbf{G})=\int_{\Omega} d \mathbf{r} e^{-i(\mathbf{Q}+\mathbf{G}) \mathbf{r}} \phi_{n \mathbf{K}}^{*}(\mathbf{r}) \phi_{n \mathbf{K}+\mathbf{Q}}(\mathbf{r}),
$$

where $\mathbf{Q}$ is the momentum transfer vector parallel to the $x-y$ plane, $\mathbf{G}=\left(\mathbf{G}_{\|}, G_{z}\right)$ are $3 D$ reciprocal lattice vectors, and $\mathbf{r}=(\boldsymbol{\rho}, z)$ is a $3 D$ position vector. Integration in (31) is performed over the normalization volume $\Omega=S \times L$, where $S$ is the normalization surface and $L$ is the superlattice constant in the $z$ direction (separation between graphene layers is superlattice arrangement). Plane wave expansion of the wave function has the form

$$
\phi_{n \mathbf{K}}(\boldsymbol{\rho}, z)=\frac{1}{\sqrt{\Omega}} e^{i \mathbf{K} \boldsymbol{\rho}} \sum_{\mathbf{G}} C_{n \mathbf{K}}(\mathbf{G}) e^{i \mathbf{G r}},
$$

where the coefficients $C_{n \mathbf{K}}$ are obtained by solving the local density approximation-Kohn Sham (LDA-KS) equations self consistently as will be discussed below. However, this straightforward calculation of graphene response functions $R(\mathbf{Q}, \omega)$ is not sufficient if we want to investigate the hybridization between the Dirac plasmon and Fuchs-Kliewer (FK) phonons at dielectric surfaces. Namely, due to the very low energy of FK phonons $(\sim 50 \mathrm{meV})$ the crossing of their dispersion relations with Dirac plasmon occurs for very small wave vectors $(Q<0.001$ a.u.). On the other hand even for very dense $K$-point mesh sampling, as for example $601 \times$ $601 \times 1$ used in this calculation, the minimum transfer wave vector $Q$ which can be reached (e.g., $Q=0.0026$ a.u. $^{-1}$ in this calculation) is still bigger than the FK phonon-Dirac plasmon crossing wave vector. Therefore we have to find the way how to calculate $R(\mathbf{Q}, \omega)$ for a denser $Q$-point mesh in the optical $Q \approx 0$ limit. One possible way is that instead of calculating response function $R^{0}(\mathbf{Q}, \omega)$ we calculate the optical $(Q=0)$ conductivity $\sigma(\omega)$. The optical conductivity in graphene can be written as [24]

$$
\sigma(\omega)=\sigma^{\text {intra }}(\omega)+\sigma^{\text {inter }}(\omega),
$$

where

$$
\sigma^{\text {intra }}(\omega)=\frac{i \rho_{0}}{\omega+i \eta_{\text {intra }}}
$$


is intraband or Drude conductivity and where

$$
\rho_{0}=-\frac{2}{\Omega} \sum_{\mathbf{K}, n} \frac{\partial f_{n}^{i}(\mathbf{K})}{\partial E_{n}(\mathbf{K})}\left|j_{n \mathbf{K}, n \mathbf{K}}^{x}(\mathbf{G}=0)\right|^{2}
$$

represents the effective number of charge carriers. The interband conductivity is

$$
\begin{aligned}
\sigma^{\text {inter }}(\omega)= & \frac{-2 i}{\omega \Omega} \sum_{\mathbf{K}, n \neq m} \frac{\hbar \omega}{E_{n}(\mathbf{K})-E_{m}(\mathbf{K})} \\
& \times \frac{f_{n}^{i}(\mathbf{K})-f_{m}^{i}(\mathbf{K})}{\hbar \omega+i \eta_{\text {inter }}+E_{n}(\mathbf{K})-E_{m}(\mathbf{K})} \\
& \times j_{n \mathbf{K}, m \mathbf{K}}^{x}(\mathbf{G}=0)\left[j_{n \mathbf{K}, m \mathbf{K}}^{x}\left(\mathbf{G}^{\prime}=0\right)\right]^{*},
\end{aligned}
$$

where the current vertices are given by

$$
j_{n \mathbf{K}, m \mathbf{K}+\mathbf{Q}}^{\mu}(\mathbf{G})=\int_{\Omega} d \mathbf{r} e^{-i(\mathbf{Q}+\mathbf{G}) \mathbf{r}} j_{n \mathbf{K}, m \mathbf{K}+\mathbf{Q}}^{\mu}(\mathbf{r}),
$$

and

$$
\begin{aligned}
j_{n \mathbf{K}, m \mathbf{K}+\mathbf{Q}}^{\mu}(\mathbf{r})= & \frac{\hbar e}{2 i m}\left\{\phi_{n \mathbf{K}}^{*}(\mathbf{r}) \partial_{\mu} \phi_{m \mathbf{K}+\mathbf{Q}}(\mathbf{r})\right. \\
& \left.-\left[\partial_{\mu} \phi_{n \mathbf{K}}^{*}(\mathbf{r})\right] \phi_{m \mathbf{K}+\mathbf{Q}}(\mathbf{r})\right\} .
\end{aligned}
$$

In the optical $Q \approx 0$ limit the independent electron response function can be written in terms of optical conductivities (32) as [40]

$$
R^{0}(\mathbf{Q} \approx 0, \omega)=L \frac{Q^{2}}{i \omega} \sigma(\omega) .
$$

Finally, the RPA or screened response function $R(\mathbf{Q}, \omega)$ can be obtained from (38) using (15).

In the calculation of Sec. V we shall assume the graphene response to be isotropic in the small $(\mathbf{Q}, \omega)$ limit. This means that the graphene response functions and the corresponding surface excitation functions are functions of $Q$ and not of $\mathbf{Q}$.

In order to explore what are the limitations of simple analytical approaches the results obtained using ab initio conductivity [(32),(33),(35)] will be compared with the results obtained using the conductivity obtained in the Dirac-cone approximation, i.e., by treating the $\pi$ electron bands as entirely conical. In the limit of zero temperature the Drude conductivity in Dirac-cone approximation can be written as [41]

$$
\sigma^{\text {intra }}(\omega)=i \frac{E_{F}}{\left(\pi \omega+i \eta_{\text {intra }}\right)},
$$

while the interband contribution is given by [41]

$$
\sigma^{\text {inter }}(\omega)=\frac{1}{4}\left[\Theta\left(\omega-2 E_{F}\right)-\frac{i}{\pi} \ln \left|\frac{2 E_{F}+\omega+\eta_{\text {inter }}}{2 E_{F}-\omega-\eta_{\text {inter }}}\right|\right],
$$

where $\Theta(\omega)$ is a Heaviside step function.

\section{A. Computational details}

The first part of the calculation consists of determining the KS ground state of the single layer graphene and the corresponding wave functions $\phi_{n \mathbf{K}}(\rho, z)$ and energies $E_{n}(\mathbf{K})$. For graphene unit cell constant we use the experimental value of $a=4.651$ a.u. [42], and superlattice unit cell constant (separation of graphene layers) is $L=5 a$. For calculating KS wave functions and energies we use a plane-wave selfconsistent field DFT code (PWSCF) within the QUANTUM ESPRESSO (QE) package [43]. The core-electron interaction was approximated by the norm-conserving pseudopotentials [44] and the exchange correlation (XC) potential by the Perdew-Zunger local density approximation (LDA) [45]. To calculate the ground state electronic density we use $21 \times 21 \times$ 1 Monkhorst-Pack $K$-point mesh [46] of the first Brillouin zone (BZ) and for the plane-wave cutoff energy we choose $50 \mathrm{Ry}$. The second part of calculation consists of determining the independent electron response function (30) and conductivity [(32)-(35)]. In order to achieve better resolution in the long wavelength $(Q \approx 0)$ and low energy $(\omega \approx 0)$ limit the response function [(30),(31)] and conductivity [(32)-(37)] are evaluated from the wave functions $\phi_{n \mathbf{K}}(\mathbf{r})$ and energies $E_{n}(\mathbf{K})$ calculated for the $601 \times 601 \times 1$ Monkhorst-Pack $K$-point mesh which corresponds to $361801 K$ points in the first Brillouin zone (1 BZ). Band summations $(n, m)$ in (30), (34), and (35) are performed over 30 bands. In the calculation we use two kinds of damping parameters: $\eta_{\text {intra }}=10 \mathrm{meV}$ for transitions within the same bands $(n \leftrightarrow n)$ and $\eta_{\text {inter }}=50 \mathrm{meV}$ for transitions between different bands $(n \leftrightarrow m)$. For bulk $\mathrm{SiO}_{2}$ dielectric function given by (28) we use the following parameters: $\epsilon_{0}=3.9, \epsilon_{i}=3.05, \epsilon_{\infty}=2.5, \omega_{T O 1}=55.6 \mathrm{meV}$, $\omega_{T O 2}=138.1 \mathrm{meV}, \gamma_{T O 1}=5.368 \mathrm{meV}$, and $\gamma_{T O 2}=8.947$ meV taken from Ref. [47]. For the gap between graphene and the $\mathrm{SiO}_{2}$ surface, we take $h=4 \AA$ [7.55 a.u.] [48].

\section{RESULTS FOR GRAPHENE MONOLAYERS $\mathrm{ON} \mathrm{SiO}_{2} \mathrm{SUBSTRATES}$}

Theoretical expressions derived in Sec. II (and in Appendix) are quite general, i.e., are valid for any pair of crystal slabs described by their response functions, while the corresponding surface excitation functions derived in Sec. III are valid for any 2D adsorbed monolayer on any dielectric substrate. In this section we shall apply these results to calculate reactive and dissipative response of various combinations of slabs consisting of graphene monolayers with variable doping on semi-infinite $(\Delta \rightarrow \infty) \mathrm{SiO}_{2}$ substrate, using the dynamical surface response functions of these materials given in Sec. III. Extension to the case of dielectric slabs of finite thickness does not change the main features in the results, however it causes the appearance of many different surface phononic modes which could disturb the interpretation of results, so we rather use semi-infinite dielectrics.

Before proceeding with the detailed calculations a few general comments are in order. For very large separations $a>10000 \AA$ the long-wavelength $\left(Q<10^{-4}\right.$ a.u. $)$ and the low energy $\left[\omega_{p} \approx 10 \mathrm{meV}(T \approx 100 \mathrm{~K})\right]$ Dirac plasmon polaritons and soft $\pi^{*} \rightarrow \pi^{*}$ electron-hole transitions $\left[\omega_{\pi^{*} \rightarrow \pi^{*}} \approx 1 \mathrm{meV}(T \approx 10 \mathrm{~K})\right]$ become dominant contributions to Casimir energy or dissipated power. Then the edge of the intraband $\pi^{*} \rightarrow \pi^{*}$ electron-hole transitions and hence frequency and broadening of plasmon polariton become 


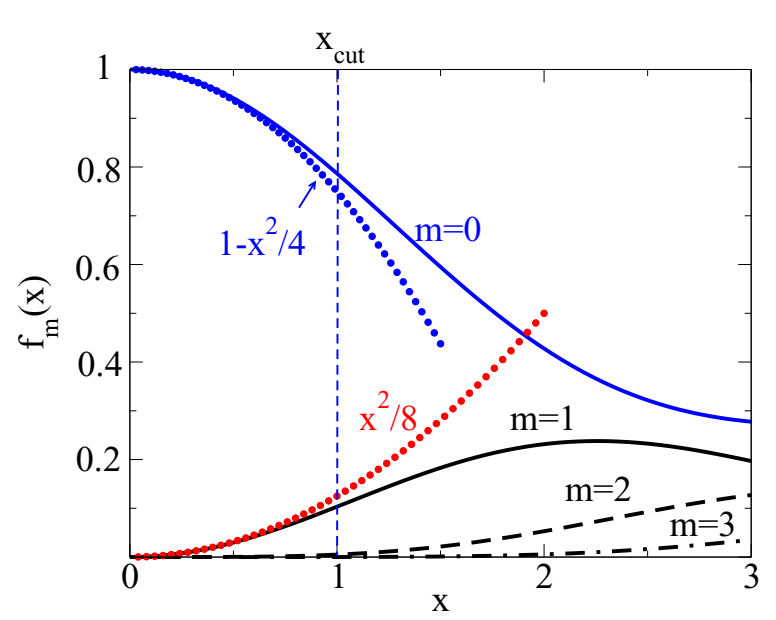

FIG. 4. Function $f_{m}(x)$ for $m=0$ (blue solid line), $m=1$ (black solid line), $m=2$ (black dashed line), and $m=3$ (black dasheddotted line). Vertical dashed line denotes the maximum argument $x_{c u t}$ defined by parameters $\left(a\right.$ and $\left.\rho_{0}\right)$ used in the calculation.

temperature dependent, so that Casimir energy at such large separations generally strongly depends on temperature, as already explored in Ref. [23]. However, for smaller separations studied here $(a<1000 \AA)$ the relevant Dirac plasmon and intraband or interband electron-hole excitation energy scale is $\omega_{\pi}, \omega_{p}>100 \mathrm{meV}(T>1000 \mathrm{~K})$ such that the $\mathrm{vdW}$ force becomes less sensitive to temperature. Therefore, even though the derived expressions for van der Waals and dissipated power (3) and (12), respectively, include temperature dependence, in the systems studied here, inclusion of finite temperature leads to practically no effects, therefore all results will be reported for $T=0$. The dependence of these two physical properties on the two parameters, the distance between the slabs $a$ and the oscillation amplitude $\rho_{0}$, can be analyzed if we recognize in the expressions (3) and (12) the function

$$
f_{m}(x)=\int_{0}^{2 \pi} \frac{d \phi}{2 \pi} J_{m}^{2}(x \cos \phi),
$$

which is possible because of the assumed isotropy of graphene response. The function $f_{m}(x)$ is shown in Fig. 4 for the first four $m$ 's, where $x=Q \rho_{0}$. Another important factor in (3) and (12) is $e^{-2 Q a}$ which defines the cutoff wave vector $Q_{c}$, depending on the slab separation $a$. The separations we shall consider in this calculation are $a=10-50 \mathrm{~nm}$ which defines the cutoff wave vector $Q_{c} \approx 0.05$ a.u.. On the other hand, the amplitudes which will be considered are $\rho_{0} \approx 0.1-1 \mathrm{~nm}$. This finally provides the maximum argument $Q$ of the functions (41) which is $x_{\text {cut }} \approx 1$. From Fig. 4 is obvious that up to $x_{\text {cut }}$ only the $m=0$ and $m=1$ terms will contribute. Moreover, for $x<x_{\text {cut }}$ the Bessels functions can be approximated as $J_{0} \approx 1-\frac{x^{2}}{4}$ and $J_{m}(x) \approx \frac{x^{m}}{2^{m} m !} ; m>1$ and therefore

$$
f_{0} \approx 1-\frac{x^{2}}{4} ; \quad f_{1}(x) \approx \frac{x^{2}}{8}
$$

In Fig. 4 we see that approximation (42) is valid almost up to $x_{\text {cut }}$.

\section{A. Spectra of coupled modes}

In this section we shall first discuss the spectra of coupled plasmon/phonon excitations in one and two graphene/ $/ \mathrm{SiO}_{2}$ slabs separated by distance $a$ in order to understand the dominant dissipation mechanisms. Figure 5(a) shows the spectrum of surface excitations $S(Q, \omega)=-\operatorname{Im} D(Q, \omega)$ in graphene $(200 \mathrm{meV}) / \mathrm{SiO}_{2}$ slab (as shown in Fig. 2) and Fig. 5(b) in the system which consists of two graphene/ $/ \mathrm{SiO}_{2}$ slabs (as shown in Fig. 1) separated by distance $a=5 \mathrm{~nm}$. In the long-wavelength limit the $\mathrm{SiO}_{2}$ surface supports two surface polar (FK) TO phonons with flat dispersions and the doped graphene contains a Dirac plasmon with square root dispersion. Coupling between these modes results in three branches, as shown in Fig. 5(a). For larger $Q$ the first and second flat branches are phononlike, i.e., their induced electrical fields mostly come from polarization modes on the dielectric surface. On the other hand, the third square root branch is plasmonlike, i.e., its induced electrical field mostly comes from charge density oscillations localized in the graphene layer. However, in the $Q \rightarrow 0$ limit the strong hybridization (avoided crossings) between these modes occur and they possess mixed plasmon-phonon character. When another slab is brought in the vicinity the three modes in each slab interact which results in the mode splitting and formation of six coupled modes as shown in Fig. 5(b). Figure 5(c) shows the spectrum of surface excitations in the graphene $(0 \mathrm{meV}) / \mathrm{SiO}_{2}$ slab. Because the pristine graphene does not support Dirac plasmon the spectrum consist just of two weak phonon branches $\omega_{T O 1}$ and $\omega_{T O 2}$ damped by $\pi \rightarrow \pi^{*}$ excitations. The spectrum of surface excitations in two equal graphene $(0 \mathrm{meV}) / \mathrm{SiO}_{2}$ slabs separated by $5 \mathrm{~nm}$ (not shown here) is very similar to the one shown in Fig. 5(c) which indicates weak interaction between phonons in the two slabs. This could be the consequence of strong screening of FK phonons by graphene adlayers which reduces the range of their induced electrical field. Figure 5(d) shows the spectrum in the system which consists of two different slabs, graphene $(0 \mathrm{meV}) / \mathrm{SiO}_{2}$ and graphene $(200 \mathrm{meV}) / \mathrm{SiO}_{2}$, separated by $5 \mathrm{~nm}$. One can notice interesting hybridization between the Dirac plasmon and two phonons in one slab and two phonons in another slab giving five branches. In the next section we shall explore how particular plasmon-phonon modes contribute to the dissipated power in two oscillating slabs.

\section{B. Modification of van der Waals force}

Van der Waals energy and attractive force are usually calculated and measured for static objects. Here we show how their relative oscillating motion can reduce this attraction, which can be relevant not only from the theoretical standpoint but also in some experimental situations and applications. This phenomenon is present also in the case of parallel motion, as shown in Appendix, but this situation would be more difficult to realize in practice.

Making use of the approximation (42) for the lowest order terms of the functions $f_{0}$ and $f_{1}$ given by (41) we can rewrite 

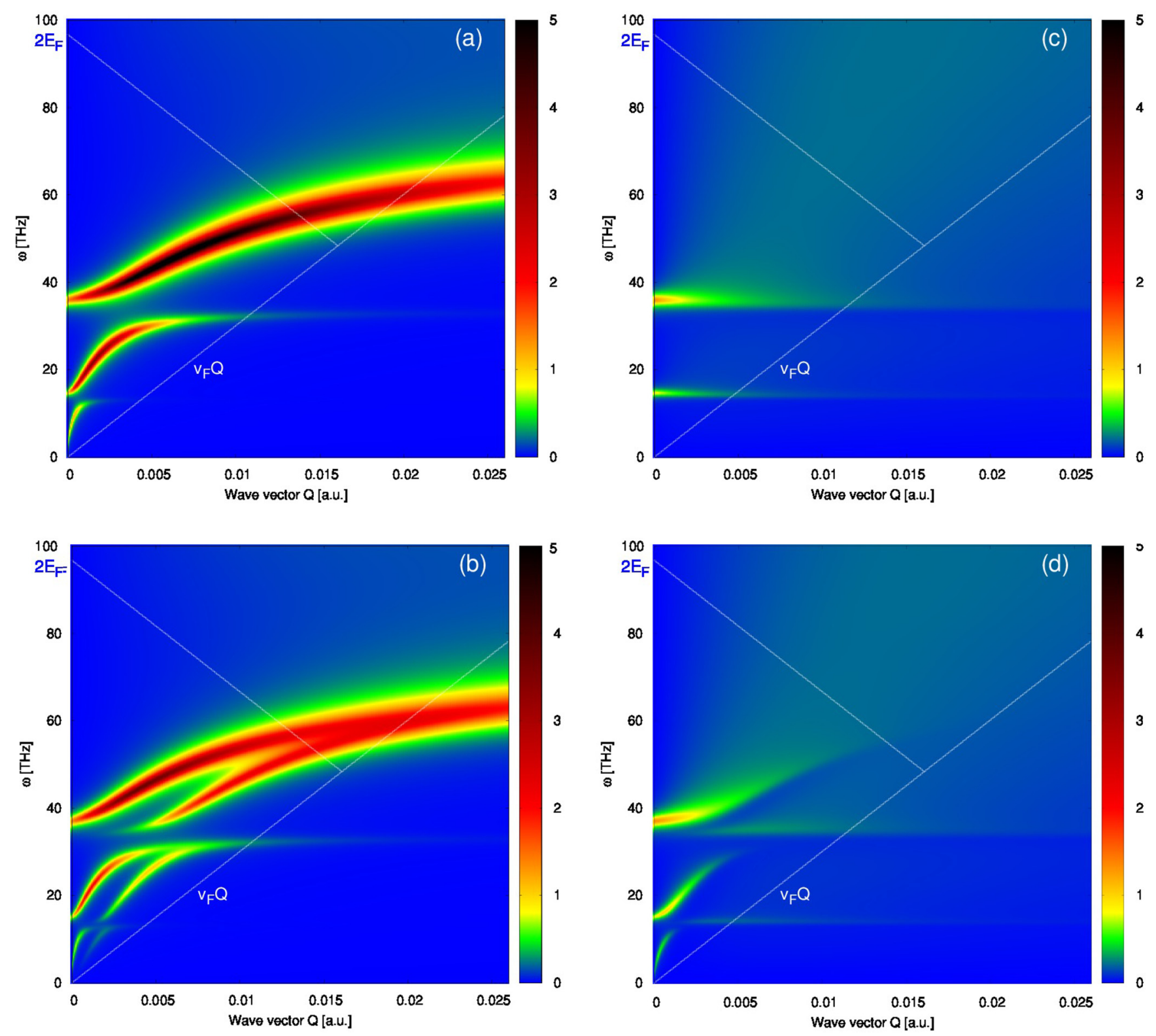

FIG. 5. The spectra of surface excitations in (a) graphene (200 meV)/SiO $\mathrm{S}_{2}$ single slab (as shown in Fig. 2), (b) in the system consisting of two equal graphene $(200 \mathrm{meV}) / \mathrm{SiO}_{2}$ slabs, (as shown in Fig. 1) separated by distance $5 \mathrm{~nm}$, (c) single graphene $(0 \mathrm{meV}) / \mathrm{SiO}{ }_{2} \mathrm{slab}$, and $(\mathrm{d})$ in the system consisting of two unequal slabs, graphene $(200 \mathrm{meV}) / \mathrm{SiO}_{2}$ and graphene $(0 \mathrm{meV}) / \mathrm{SiO}_{2}$, separated by distance $5 \mathrm{~nm}$.

the expression (3) for the van der Waals energy as

$$
\begin{aligned}
E_{c}(a)= & \frac{\hbar}{2} \int \frac{Q d Q}{2 \pi} \int_{-\infty}^{\infty} \frac{d \omega}{2 \pi} \\
& \times\left\{\left[1-\frac{Q^{2} \rho_{0}^{2}}{4}\right] A(Q, \omega, \omega)\right. \\
& \left.+\frac{1}{4} Q^{2} \rho_{0}^{2} A\left(Q, \omega, \omega-\omega_{0}\right)\right\},
\end{aligned}
$$

where $A$ is given by (A17) and (A18). In the $T \rightarrow 0$ limit and neglecting higher order terms $A$ reduces to

$$
\begin{aligned}
& A\left(Q, \omega, \omega^{\prime}\right) \\
& \quad=e^{-2 Q a} \operatorname{sgn} \omega\left\{\operatorname{Im} D_{1}(Q, \omega) \operatorname{Re} D_{2}\left(Q, \omega^{\prime}\right)+(1 \leftrightarrow 2)\right\} .
\end{aligned}
$$

We see that for $\rho_{0} \rightarrow 0$ the van der Waals energy reduces to the standard result for the static case, and for $\rho_{0} \neq 0$ and $\omega_{0} \neq$ 0 the lowest order corrections scale with $\rho_{0}^{2}$. From (43), and also from (44), we see that the slab separation $a$ (because of exponential factor $e^{-2 Q a}$ ) reduces the wave vector range to $Q<1 / 2 a$.

Figure 6 shows van der Waals energies $E_{c}$ of two variously doped, unsupported full conductivity (32)-(35) graphenes as functions of the driving frequency $\omega_{0}$. The driving amplitude is $\rho_{0}=20 \mathrm{~nm}$ and separation between slabs is $a=10 \mathrm{~nm}$. For the case of two heavily and equally doped graphenes $1-1 \mathrm{eV}$ (thick black solid line) the 'static' $\left(\omega_{0}=0\right)$ van der Waals energy is the largest in comparison with other doping combinations. This is reasonable considering that then except of $\pi$ and $\pi+\sigma$ plasmons (and corresponding electron-hole excitations) the graphenes support strong Dirac plasmons 


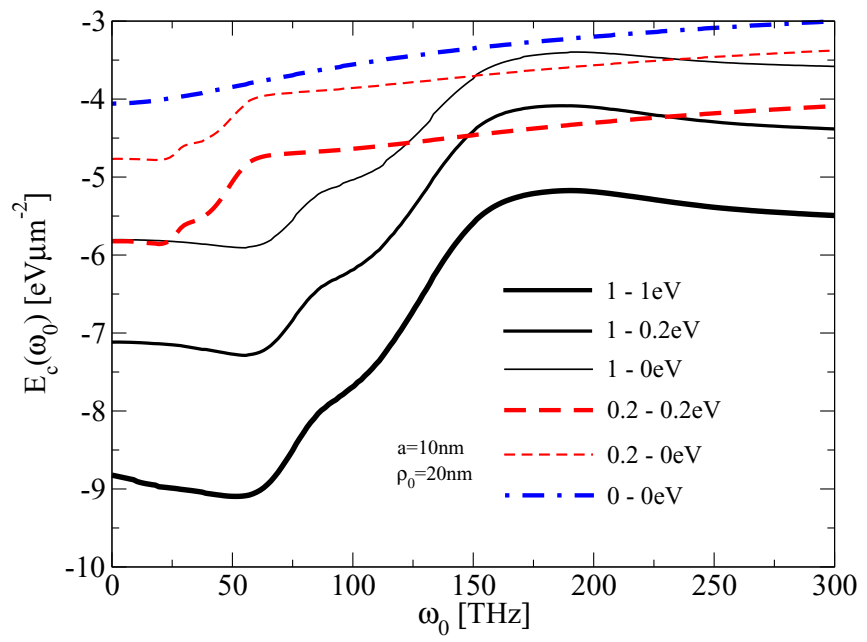

FIG. 6. Van der Waals energies $E_{c}$ of two variously doped, unsupported full conductivity (32)-(35) graphenes as functions of driving frequency $\omega_{0}$. The left-right graphene dopings are $1-1 \mathrm{eV}$ (thick black solid), 1-0.2 eV (black solid), 1-1 eV (thin black solid), $0.2-0.2 \mathrm{eV}$ (red dashed), $0.2-0 \mathrm{eV}$ (thin red dashed), $0-0 \mathrm{eV}$ (blue dashed-dotted), as also denoted in the figure. Separation between graphenes is $a=10 \mathrm{~nm}$ and oscillating amplitude is $\rho_{0}=20 \mathrm{~nm}$.

which are all in resonance. Therefore, the charge density fluctuation in one slab $\operatorname{Im} D_{1}(\omega)$ resonantly induces electrical field in another slab $\operatorname{Re} D_{2}(\omega)$ to which it couples, and vice versa. As the driving frequency $\omega_{0}$ increases the fluctuation and the induced field do not match any more, i.e., $\operatorname{Im} D_{1}(\omega)$ and $\operatorname{Re} D_{2}\left(\omega+n \omega_{0}\right)$ become Doppler shifted and vdW energy is expected to decrease. However, the vdW energy first exhibits a wide plateau until $\omega_{0}<50 \mathrm{THz}$. We performed a separate vdW energy calculation for two unsupported Drude [(32),(33)] graphenes (not shown here) and noticed that it shows the same features as presented in Fig. 6. This suggests that Dirac plasmons are responsible for all characteristic features in vdW energy (for larger dopings). Therefore, the plateau arises probably because the Dirac plasmon fluctuation in one slab, e.g., at $\omega_{p}$, can be efficiently screened by induced plasmon field in another slab which is not necessarily at the same frequency $\omega_{p}$. Moreover, graphene, regardless of doping, exhibits perfect screening $\operatorname{Re} D(Q \approx 0, \omega \approx 0) \approx-1$ [38] causing that the static point charge feels image potential. This causes that $E_{c}$ shows almost identical plateau for the case of differently doped graphenes $1-0.2 \mathrm{eV}$ (black solid line) and $1-0 \mathrm{eV}$ (thin black solid line). As the doping difference increases plateau energy decreases which is reasonable because of plasmon resonance breakdown. For larger $\omega_{0}>50 \mathrm{THz}$ the Dirac plasmon in one slab does not match any more the perfect screening regime in another one, resulting in a rapid decrease or weakening of $\mathrm{vdW}$ energy. In the case of weakly doped graphenes, such as the combinations $0.2-0.2 \mathrm{eV}$ (red dashed line) and $0.2-0 \mathrm{eV}$ (thin red dashed lines), the 'static' $\omega_{0} \approx 0$ van der Waals energy reduces in comparison with the heavy doping (combinations with $1 \mathrm{eV}$ ) cases. This is reasonable considering that Dirac plasmon spectral weight decreases with doping. Additionally, it can be noted that for lower doping the vdW plateau shifts to $\omega_{0}<25 \mathrm{THz}$. This is because the

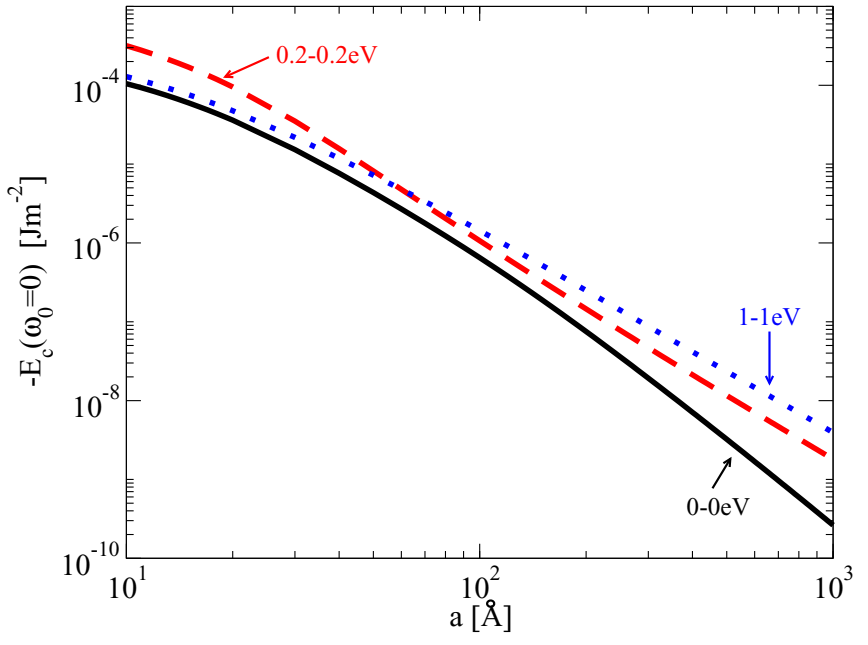

FIG. 7. Static $\omega_{0}=0$ van der Waals energy $E_{c}$ of two unsupported full conductivity [(32)-(35)] graphenes as a function of separation $a$. The left-right graphene dopings are $0-0 \mathrm{eV}$ (black solid), $0.2-0.2 \mathrm{eV}$ (red dashed), $1-1 \mathrm{eV}$ (blue dotted), as also denoted in the figure. The dopings $E_{F}=0.2 \mathrm{eV}$ and $1 \mathrm{eV}$ correspond to $\pi^{*}$ electron densities $2.9 \times 10^{12} \mathrm{~cm}^{-2}$ and $7.3 \times 10^{13} \mathrm{~cm}^{-2}$, respectively.

perfect screening frequency region can be roughly estimated as $\operatorname{Re} D\left(\omega<\omega_{p}\right) \approx-1$, so, as the plasmon energy decreases the frequency interval within which fluctuations are perfectly screened becomes narrower. It is interesting to notice that for some frequencies (e.g., $\omega_{0}>100 \mathrm{THz}$ ) the resonant but low doping vdW energy (e.g., $0.2-0.2 \mathrm{eV}$ case) overcomes the heavily doped but off resonance vdW energy (such as the cases $1-0.2 \mathrm{eV}$ and $1-0 \mathrm{eV}$ ). The static $\omega_{0}=0 \mathrm{vdW}$ energy of pristine graphenes $0-0 \mathrm{eV}$ (blue dashed dotted line) is the weakest and shows smooth decreasing, almost linear behavior. In this case there are no Dirac plasmons in the graphenes spectra. Therefore, only resonant coupling between $\pi \rightarrow \pi^{*}$ electron-hole excitations, $\pi$ and $\pi+\sigma$ plasmons contribute to the vdW energy. As the frequency $\omega_{0}$ increases the overlap between these electronic excitations decreases causing smooth and linear vdW energy weakening. The same linear behavior (for $\omega_{0}>50 \mathrm{THz}$ ) can be noticed for doping combinations $0.2-0.2 \mathrm{eV}$ and $0.2-0 \mathrm{eV}$ which proves that for lower dopings the dominant $\mathrm{vdW}$ energy weakening mechanism becomes off-resonant coupling between $\pi \rightarrow \pi^{*}$ electron-hole excitations, $\pi$ and $\pi+\sigma$ plasmons.

It should be noted here that such designed (graphene based) slabs might enable modification of attraction between slabs, e.g., controlled the binding energy between two slabs. For example, two heavily doped graphenes (1-1 eV case in Fig. 6) are strongly bound, however binding energy between pristine graphenes $(0-0 \mathrm{eV}$ case achieved, e.g., simply by electrostatic gating) is reduced more than twice. Moreover, for larger $\omega_{0}$ (and fixed doping) the dynamical binding energy is substantially reduced, leading to decoupling of two slabs, and vice versa, their recoupling by reducing the driving frequency.

Figure 7 shows static $\left(\omega_{0}=0\right)$ van der Waals energy of two unsupported graphenes as a function of separation $a$ for various symmetrical dopings $0-0 \mathrm{eV}$ (black solid), $200-200 \mathrm{meV}$ (red dashed), and 1-1 eV (blue dotted). As expected, the pristine graphenes show the lowest vdW energy, while in doped 

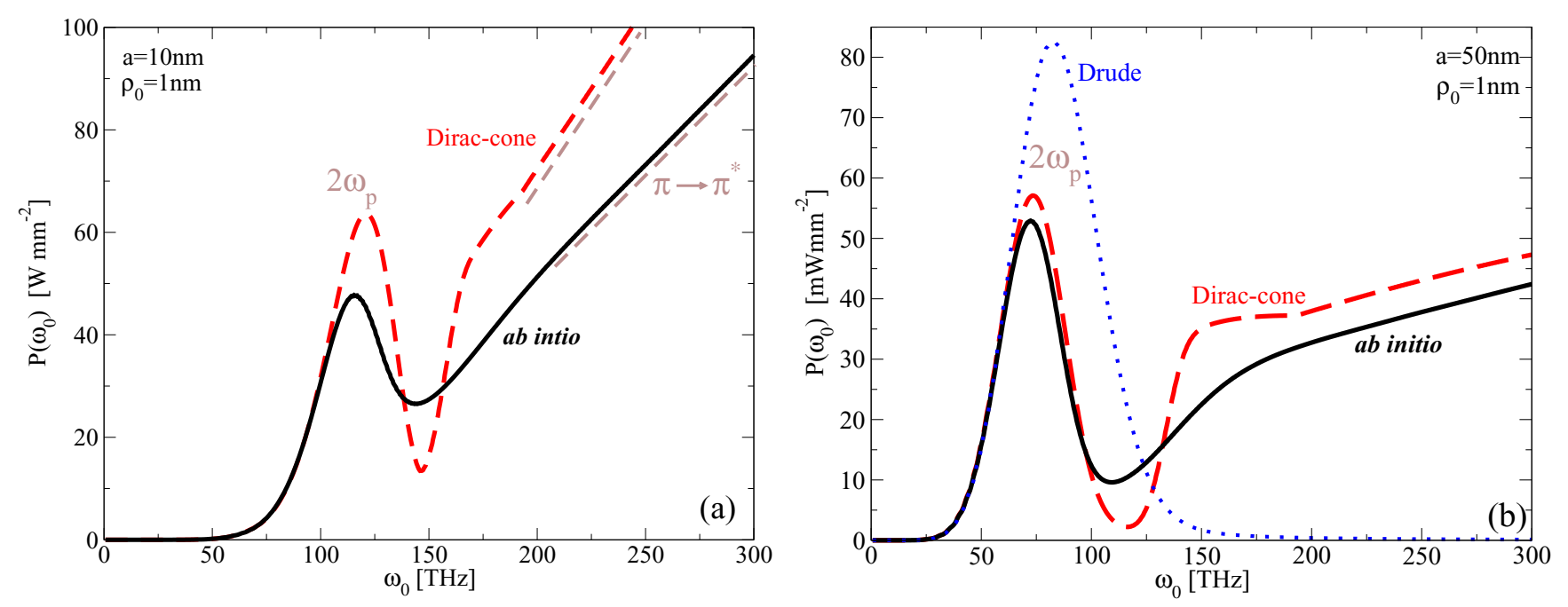

FIG. 8. Dissipated power in two oscillating unsupported graphenes obtained using ab initio conductivity [(32),(33),(35)] (black solid line), Dirac-cone approximation conductivity [(39),(40)] (red-dashed line), and using Dirac-Drude approximation conductivity (39) (blue-dotted line). The separations between slabs and oscillation amplitudes are (a) $a=10 \mathrm{~nm}, \rho_{0}=1 \mathrm{~nm}$, (b) $a=50 \mathrm{~nm}, \rho_{0}=1 \mathrm{~nm}$. Both graphenes are doped such that $E_{F 1}=E_{F 2}=200 \mathrm{meV}$.

graphenes, which support additional plasmon, vdW energy is larger. However, the vdW energies in doped graphenes show counterintuitive crossover behavior at about $a=50 \AA$, such that, for $a<50 \AA$, graphenes with smaller doping show larger $\mathrm{vdW}$ binding energy than graphenes with larger doping. This is probably because large doping increases forbidden electronhole excitation areas, i.e., reduces electron-hole contributions to vdW energy especially important for smaller separations. For larger separations Dirac plasmons represent the dominant contribution to vdW energy, so that graphenes with larger doping (and stronger Dirac plasmon) show stronger vdW binding energy. Finally, this can be clearly seen for very large separations (e.g., at $a \approx 10^{3} \AA$ ), where vdW binding between doped graphenes is an order of magnitude stronger than $\mathrm{vdW}$ binding between pristine graphenes, which do not support Dirac plasmon. Similar qualitative trend (increase of vdW energy with doping) and the same quantitative estimation of the $\mathrm{vdW}$ binding energies is documented in many papers $[8,20]$, though there are also some qualitative disagreements. For example, in Fig. 2 of Ref. [20] the vdW energy vs separation $a$ is more linear and also without crossover. The disagreement is probably because the Dirac-cone approach does not provide screening accurately enough (real part of dynamical response) which is especially important for accurate estimation of reactive $\mathrm{vdW}$ force. On the other hand, the dissipative, friction force is more sensitive to the imaginary part of dynamical response or to the real part of dynamical conductivity where the Dirac-cone approach is more successful and hence provides better agreement with ab initio friction, especially for smaller frequencies $\omega_{0}$ (which corresponds to large separations $a$ ), as will be discussed in the next section.

\section{Dissipated power-Comparison with Dirac-cone analytical model}

In order to explore how the details of realistic graphene band structure influence the dissipated power, i.e., what are the limitations of simple Dirac-cone approximation, here we compare the results of these two models. Figure 8 shows the dissipated power in two oscillating unsupported graphenes obtained using ab initio conductivity [(32),(33),(35)] (black solid line) and Dirac-cone approximation conductivity [(39),(40)] (red-dashed line). The separations between slabs and oscillation amplitudes are denoted on the graphs. Both graphenes are doped such that $E_{F 1}=E_{F 2}=200 \mathrm{meV}$.

As can be noticed in Fig. 8(a) the dissipated power consists of peak at $2 \omega_{p}$ which comes from the excitation of Dirac plasmons, and of linear behavior starting at about $200 \mathrm{THz}$ $(800 \mathrm{meV})$ (emphasized by brown lines) which is the fingerprint of interband $\pi \rightarrow \pi^{*}$ excitations. As expected, for smaller frequencies $\left(\omega_{0}<100 \mathrm{THz}[400 \mathrm{meV}]\right)$, when low energy, mostly intraband $\pi^{*} \rightarrow \pi^{*}$ excitations contribute, the two models agree perfectly. For frequencies $\omega_{0} \approx 2 \omega_{p}$ $(500 \mathrm{meV})$ the ab initio dissipation is smaller than in the Dirac cone approach. This is because the $a b$ initio model includes $\pi$ and $\pi+\sigma$ plasmons, which screen and reduce Dirac plasmon intensity [33], while conical $\pi$ band (without band banding in the K-M direction, as shown in Fig. 3) and absence of $\sigma$ bands result that there is no $\pi$ nor $\sigma$ plasmons. For larger frequencies $\omega_{0}>2 \omega_{p}$ the main dissipation contribution comes from resonant coupling between Dirac plasmons and $\pi \rightarrow \pi^{*}$ excitations. The $a b$ initio dissipation is smoother and mostly smaller which is probably due to the fact that ab initio Dirac plasmon is earlier Landau damped (broader) and weaker (due to mentioned screening mechanisms) than in the Dirac-cone approximation.

The dissipated power for larger separations $a=50 \mathrm{~nm}$ is shown in Fig. 8(b). Considering that now the modes with smaller wave vectors $Q$ and thus the Dirac plasmon with smaller energies contribute the agreement between $a b$ initio and Dirac-cone approach is better. Moreover, dissipated power calculated using simple Dirac-Drude approximation (blue dotted line), i.e., using conductivity (39), for $\omega_{0}<50 \mathrm{THz}$, shows excellent agreement with the other two 

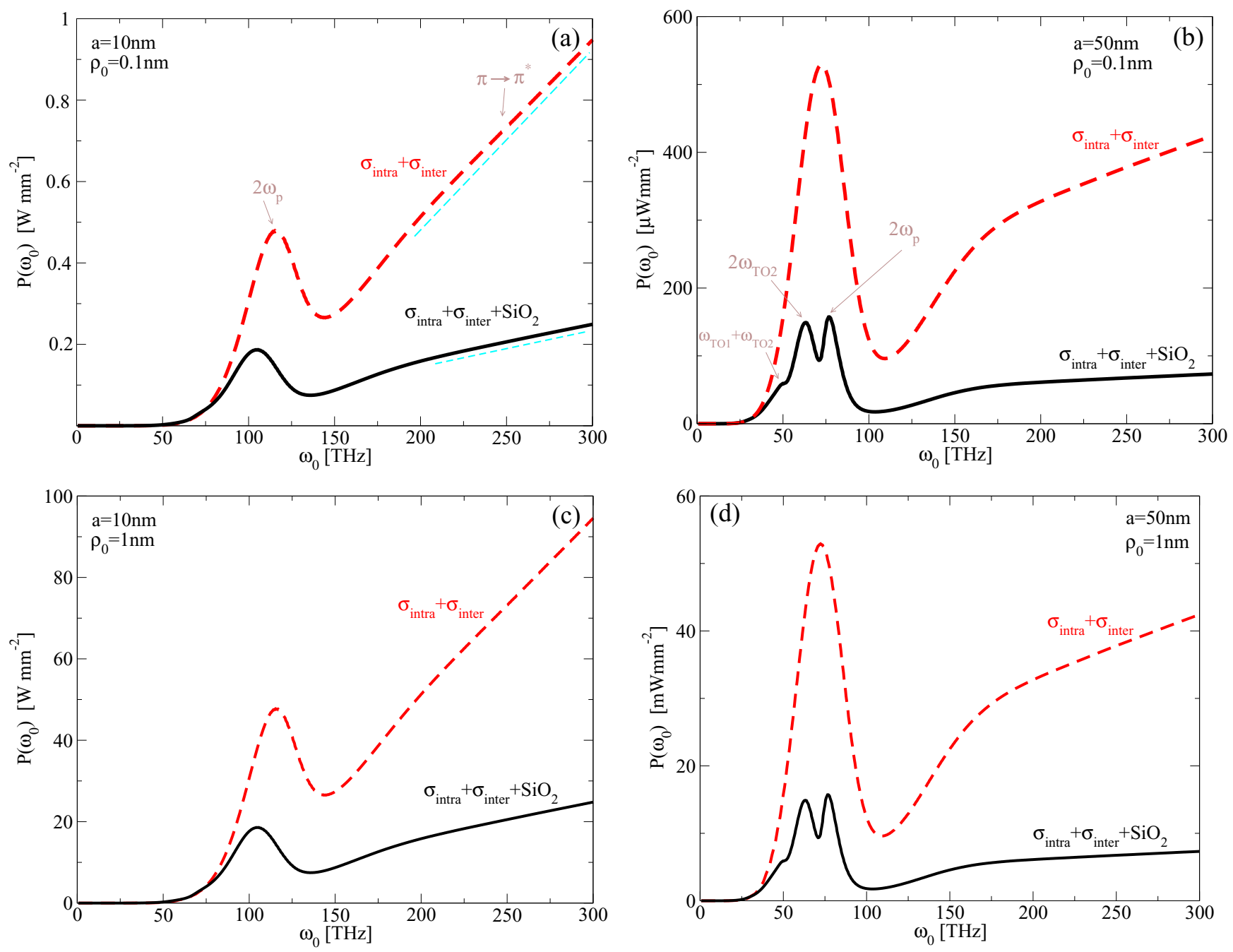

FIG. 9. Dissipated power in two oscillating unsupported ab initio conductivity [(32),(33),(35)] graphenes (red dashed line) and $a b$ initio conductivity graphenes deposited on semi-infinite $(\Delta \rightarrow \infty) \mathrm{SiO}_{2}$ substrates (black solid line). The separations between slabs and oscillation amplitudes are (a) $a=10 \mathrm{~nm}, \rho_{0}=0.1 \mathrm{~nm}$, (b) $a=50 \mathrm{~nm}, \rho_{0}=0.1 \mathrm{~nm}$, (c) $a=10 \mathrm{~nm}, \rho_{0}=1 \mathrm{~nm}$, and (d) $a=50 \mathrm{~nm}, \rho_{0}=1 \mathrm{~nm}$. Both graphenes are doped such that $E_{F 1}=E_{F 2}=200 \mathrm{meV}$.

methods. Drude dissipation shows a stronger peak which comes from the excitation of undamped Dirac plasmons, however, after inclusion of interband term (red dashed line) the plasmon peak is weakened and slightly redshifted. This is reasonable considering that $\pi \rightarrow \pi^{*}$ transitions screen the Dirac plasmon, i.e., push its dispersion toward lower energies and reduce its plasmon pole weight.

\section{Dissipated power-Substrate dependence}

In this section we shall explore how the dissipated power in two oscillating slabs depends on the conductivity model we use to describe graphene and how substrate influences the dissipated power. In order to facilitate the analysis of the results we shall again use the approximation (42). The lowest order term which contributes in (12) is $f_{1}$, and from Fig. 4 it is obvious that, for $x<x_{\text {cut }}$, the higher order terms $(m=2,3, \ldots)$ do not contribute and $f_{1}$ can be freely approximated by (42) (red dotted line). Furthermore, because the higher order processes (see Fig. 12) included in (12) weakly influence the power $P$ it can be calculated using equation (11) which includes only the lowest order process. Therefore the formula for the dissipated power can be rewritten as

$$
\begin{aligned}
P= & \frac{\hbar \omega_{0} \rho_{0}^{2}}{4 \pi} \int Q^{3} d Q e^{-2 Q a} \\
& \times \int_{0}^{\omega_{0}} \frac{d \omega}{2 \pi} \operatorname{Im} D_{1}(Q, \omega) \operatorname{Im} D_{2}\left(Q, \omega_{0}-\omega\right) .
\end{aligned}
$$

This suggests that the dissipated power, within the parameter space used in this investigation (for $x<x_{\text {cut }}$ ), behaves as $P \sim \rho_{0}^{2}$. Also Eq. (44) suggests that the resonant condition (maximum in $P$ ) will occur when the driving frequencies satisfy the condition

$$
\omega_{0}=n_{1} \omega_{i}+n_{2} \omega_{j} ; n_{1}, n_{2}=1,2,3, \ldots,
$$

where $\omega_{i}=\omega_{p}, \omega_{T O 1}$, and $\omega_{T O 2}$ are the frequencies of hybridized Dirac plasmons and TO phonons, respectively.

Figure 9 shows the dissipated power $P\left(\omega_{0}\right)$ for two oscillating unsupported graphene (no substrate) monolayers 
obtained using $a b$ initio conductivity $(32,33,35)$ (red dashed line), and also for ab initio graphenes on semi-infinite $(\Delta \rightarrow$ $\infty \mathrm{SiO}_{2}$ substrates (black solid line). Both graphene monolayers are doped so that $E_{F 1}=E_{F 2}=200 \mathrm{meV}$. In Fig. 9(a) the separation between slabs and oscillation amplitude are $a=10 \mathrm{~nm}$ and $\rho_{0}=0.1 \mathrm{~nm}$, respectively. As already discussed, $P$ for unsupported graphenes shows a peak at $2 \omega_{p}$ which comes from the resonant coupling between Dirac plasmons in two slabs, and for higher frequencies $\omega_{0}>200 \mathrm{THz}$ it shows linear behavior [emphasized in Fig. 9(a) by cyan dashed lines] due to the resonant coupling between Dirac plasmon and interband $\pi \rightarrow \pi^{*}$ excitations.

In the presence of the substrate dissipation is additionally reduced by almost a factor of three. This is because for smaller separations $(a=10 \mathrm{~nm})$ the modes with higher wave vectors (e.g., $Q \approx 0.01$ a.u.), which is in this case only the Dirac plasmon, dominantly contribute to $P$. In this wave-vector region the Dirac plasmon already has high enough frequency $(\omega \approx 60 \mathrm{THz})$ that the dynamical part of the substrate screening is not active and the substrate dielectric function can be approximated by $\epsilon_{S}(\omega) \approx \epsilon_{\infty}$. This causes the reduction of substrate screened Coulomb interaction $\tilde{v}_{Q}(\omega)=\frac{2}{1+\epsilon_{S}(\omega)} v_{Q}$ [see Eq. (18)] and then [considering Eq. (23)] reduction of the plasmon intensity, which finally causes the reduction of $P$. Reduction of the screened Coulomb interaction (18) also causes the reduction of the plasmon frequency which can also be noted.

Figure 9(b) shows the dissipated power $P\left(\omega_{0}\right)$ for the same set of parameters as in Fig. 9(a) except that the separation between slabs is increased to $a=50 \mathrm{~nm}$. As expected, from the discussion in Sec. V B, $P$ is reduced by about four orders of magnitude and plasmon peaks are shifted toward lower frequencies. The latter is also expected considering that for larger separations the modes with smaller $Q$ contribute, and here the Dirac plasmon has lower energy. We can notice qualitative difference between $P$ in Figs. 9(a) and 9(b) for the case when substrate is present (black lines). In Fig. 9(b) $P$ possesses additional structures (two additional peaks at $\omega_{T O 1}+\omega_{T O 2}$ and $\left.2 \omega_{T O 2}\right)$ which are not present in Fig. 9(a). This is because for larger $a$ the modes with smaller wave vectors (e.g., $Q \approx 0.002$ a.u.) start contributing to $P$, and this is exactly the region where plasmon/phonon hybridization occurs [as illustrated in Fig. 5(a)], so the additional peaks at $\omega_{T O 1}+\omega_{T O 2}$ and $2 \omega_{T O 2}$ represent the resonant dissipation to two phonon modes.

Figures $9(\mathrm{c})$ and $9(\mathrm{~d})$ show the dissipated power $P$ for the same parameters as in Figs. 9(a) and 9(b), respectively, except that the oscillation amplitude is increased to $\rho_{0}=1 \mathrm{~nm}$. $P$ in Figs. 9(c) and 9(d) are qualitatively the same and exactly one hundred times larger than $P$ in Figs. 9(a) and 9(b). This confirms $P \sim \rho_{0}^{2}$ behavior of the dissipated power with amplitude as predicted by Eq. (44).

\section{E. Dissipated power-Graphene doping and distance dependence}

In this section we shall explore the dissipated power for two oscillating slabs for different graphene dopings. Figure 10(a) shows the dissipated power in two oscillating graphenes deposited on semi-infinite $(\Delta \rightarrow \infty) \mathrm{SiO}_{2}$ substrates where the graphene dopings $E_{F 1}-E_{F 2}$ are 0-0 $\mathrm{meV}$ (blue thin line), $0-200 \mathrm{meV}$ (red dashed line), and 200-200 meV (black solid line). The separations between slabs and oscillation amplitude are $a=10 \mathrm{~nm}$ and $\rho_{0}=$ $0.1 \mathrm{~nm}$, respectively.

If both graphenes are doped $P$ shows the plasmon peak at about $2 \omega_{p}=100 \mathrm{THz}$, and starting at about $200 \mathrm{THz}$ it increases linearly, which is the consequence of interband $\pi \rightarrow$ $\pi^{*}$ excitations, as already observed in Fig. 9. However, if one doped graphene is replaced by pristine graphene $\left(E_{F}=\right.$ 0 ), which does not support the Dirac plasmon [as shown in Fig. 5(c)], the Dirac plasmon in doped graphene can no longer resonantly transfer energy to the Dirac plasmon in another graphene and $P$ loses the plasmon peak at $2 \omega_{p}$. However, the visible step remains (at about $75 \mathrm{THz}$ ) which is the consequence of energy transfer between Dirac plasmon in the doped graphene and $\pi \rightarrow \pi^{*}$ excitations in the undoped one. In this case (small $a$ and larger $Q$ ) phonons are still very weak and do not represent an important dissipation channel. When both graphenes are pristine the only dissipation comes from the resonant energy transfer between $\pi \rightarrow \pi^{*}$ excitations in different graphenes, resulting in the strictly linear behavior of $P$.

Figure 10(b) shows the dissipated power $P$ for the same parameters as in Fig. 10(a) except that the separation between slabs is increased to $a=50 \mathrm{~nm}$. As we have already discussed in Fig. 9(a), in this case the modes with smaller wave vectors $Q$ contribute and the dissipated power $P$ gets additional structures coming from resonant phonon excitations. For the case $E_{F 1}-E_{F 2}=200-200 \mathrm{meV}$ (black solid line) [coupling between modes in Fig. 5(a)] the dissipated power shows three peaks at $\omega_{T O 1}+\omega_{T O 2} \approx 40 \mathrm{THz}, 2 \omega_{T O 2} \approx 60 \mathrm{THz}$, and $2 \omega_{p} \approx 75 \mathrm{THz}$. For the case $E_{F 1}-E_{F 2}=0-200 \mathrm{meV}$ (red dashed line) there is a possibility for resonant coupling between two phonons in the slab with pristine graphene and three hybridized plasmon/phonon modes in the slab with doped graphene [coupling between modes in Fig. 5(a) and modes in Fig. 5(c)]. The three peaks correspond to resonant couplings at $\omega_{T O 1}+\omega_{T O 2}, 2 \omega_{T O 2}$, and $\omega_{T O 2}+\omega_{p}$, as denoted in Fig. 10(b). When both graphenes are pristine, i.e., $E_{F 1}-E_{F 2}=0-0 \mathrm{meV}$ (thin solid blue line) the dominant dissipation channels become the resonant coupling between phonons in both slabs [coupling between modes in Fig. 5(c)]. The three peaks correspond to resonant couplings at $2 \omega_{T O 1}$, $\omega_{T O 1}+\omega_{T O 2}$, and $2 \omega_{T O 2}$, as denoted in Fig. 10(b). Of course, in all three cases $P$ shows linear behavior for larger $\omega_{0}$ coming from the resonant $\pi \rightarrow \pi^{*}$ excitations in both slabs. Figures 10(c) and 10(d) show the same as Figs. 10(a) and 10(b), except that the oscillation amplitude is increased to $\rho_{0}=1 \mathrm{~nm}$. As in Figs. 9, $P$ is qualitatively similar and exactly one hundred times larger than $P$ in Figs. 10(a) and 10(b). This again confirms the $P \sim \rho_{0}^{2}$ behavior. This strong dependence of dissipated power on graphene doping suggests many opportunities for applications.

There is no simple quantitative agreement between the dissipated power $P$ for uniform and oscillatory motion. Some average uniform moving velocity in the oscillatory case can be estimated as

$$
\bar{v}=\omega_{0}\left|\rho_{0}\right| / 2 \pi \text {. }
$$



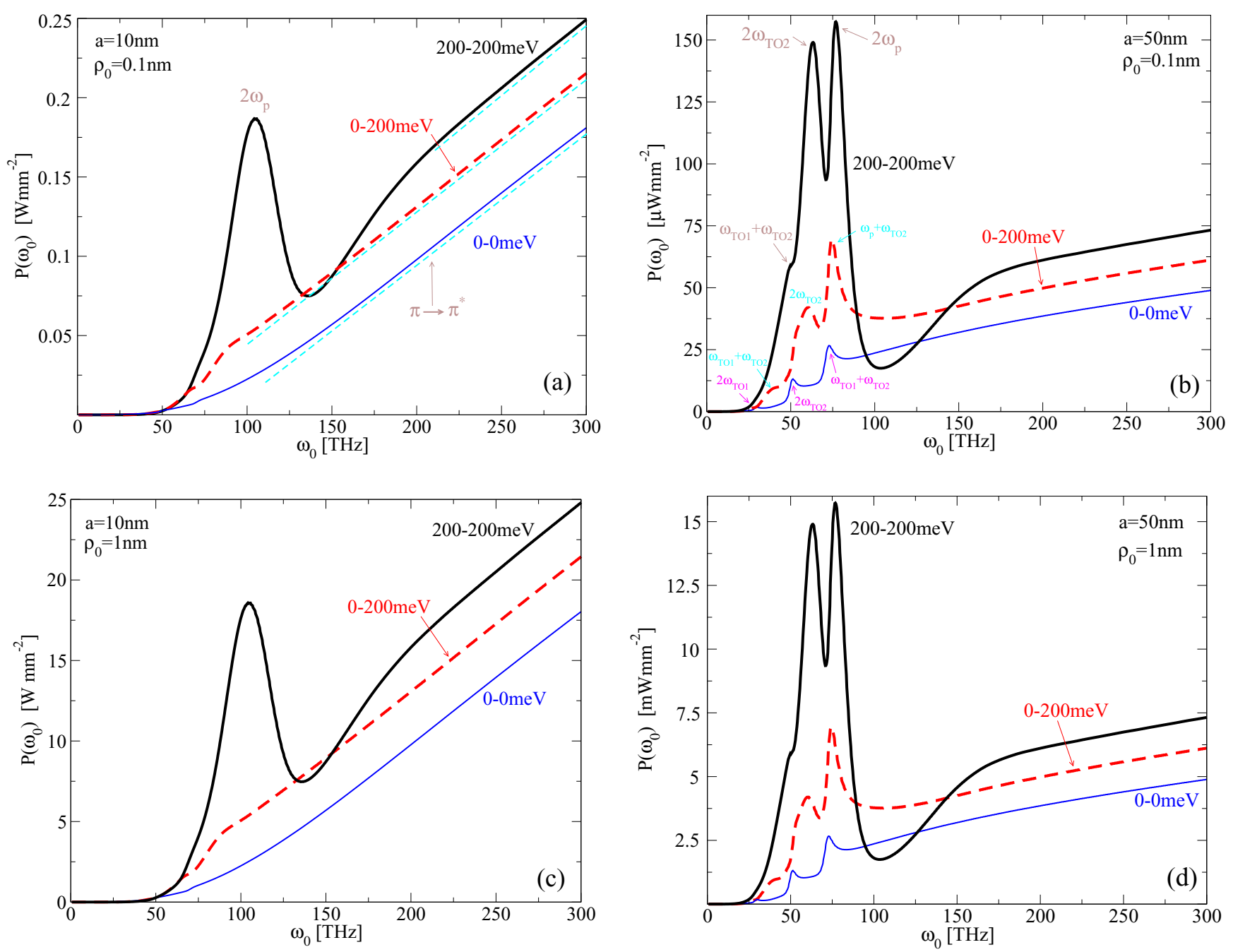

FIG. 10. Dissipated power for two oscillating graphenes deposited on semi-infinite $(\Delta \rightarrow \infty) \mathrm{SiO}_{2}$ substrates where the graphene dopings $E_{F 1}-E_{F 2}$ are 0-0 meV (blue thin line), 0-200 meV (red dashed line), and 200-200 meV (black solid line). The separations between slabs and oscillation amplitudes are (a) $a=10 \mathrm{~nm}, \rho_{0}=0.1 \mathrm{~nm}$, (b) $a=50 \mathrm{~nm}, \rho_{0}=0.1 \mathrm{~nm}$, (c) $a=10 \mathrm{~nm}, \rho_{0}=1 \mathrm{~nm}$, and (d) $a=50 \mathrm{~nm}$, $\rho_{0}=1 \mathrm{~nm}$. The graphene response is calculated using full conductivity expression [(32)-(38)].

The mechanisms of electronic mode overlaps in oscillatory and uniform motion cases are different. For example, for the oscillatory motion case the mode overlap contribution in Eq. (11) for the $m=1$ case is

$$
\int_{0}^{\omega_{0}} \operatorname{Im} D_{1}(\mathbf{Q}, \omega) \operatorname{Im} D_{2}\left(\mathbf{Q}, \omega_{0}-\omega\right) d \omega
$$

and for the uniform motion case, from Eq. (A40) it is

$$
\int_{0}^{\mathbf{Q v}} \operatorname{Im} D_{1}(\mathbf{Q}, \omega) \operatorname{Im} D_{2}(\mathbf{Q}, \mathbf{v} \mathbf{Q}-\omega) d \omega
$$

Therefore, because of the $\mathbf{v Q}-\omega$ term in (48) the acoustic modes $[\omega(Q)=\alpha Q]$ will perfectly match for $v=2 \alpha$ providing a well defined peak in the friction force, such as in the case of acoustic plasmon in Fig. 10 of Ref. [49]. On the other hand, because of the $\omega_{0}-\omega$ term in (47) the acoustic modes would match just in one $(Q, \omega)$ point, providing a dispersive peak in friction force. Exactly the opposite is valid for dispersionless modes, such as TO phonons $\left[\omega(Q)=\omega_{T o}\right]$ where $\omega_{0}-\omega$ in (47) ensures the perfect matching for $\omega_{0}=2 \omega_{T O}$, as seen in Figs. 9 and 10. Therefore, there exists only qualitative agreement which says that dissipation will be enhanced if there is some matching between particular modes, so that, e.g., 2D plasmon will generate a peak in oscillatory or in linear friction. However, the peak positions cannot be related simply by using (46).

\section{CONCLUSIONS}

In this paper we have provided a complete theoretical description of van der Waals and friction forces for two slabs in relative oscillatory motion which includes variable temperatures in two slabs, their dynamical properties, and dependence on characteristic oscillation amplitude and frequency. In the Appendix we also provide, for comparison, analogous expressions for the slabs in parallel uniform motion.

We applied this formulation to explore van der Waals and friction forces between two oscillating slabs, each consisting of atomically thick crystal (e.g., graphene) adsorbed on a dielectric substrate $\left(\mathrm{SiO}_{2}\right)$. We explore dependence of these 
forces on oscillator characteristics such as driving amplitude $\rho_{0}$ and frequency $\omega_{0}$, but also on slab separation $a$, on graphene doping $E_{F}$, and on substrate properties. We show how the spectra of coupled electronic/phononic excitations in slabs determine the energy transfer processes in this system.

We show that, in general, as the driving frequency $\omega_{0}$ increases the vdW energy first shows an unusual plateau and then decreases. We propose the idea of controlling the binding energy between two slabs by tuning the graphene dopings $E_{F i}$ and the driving frequency $\omega_{0}$. We also found a simple $\rho_{0}^{2}$ dependence of both the vdW force and dissipated power.

It is shown that for smaller frequencies $\left[\omega_{0}<100 \mathrm{THz}\right.$ $(400 \mathrm{meV})]$ dissipated power calculated using Dirac-cone conductivity shows good agreement with the ab initio results, while for larger frequencies Dirac-cone approximation overestimates $a b$ initio dissipation. However, in a realistic graphene (whose dielectric properties are calculated from first principles) this peak is strongly reduced and redshifted. We also explain why the substrate substantially reduces dissipated power peak $2 \omega_{p}$. For larger separations $a$ additional peaks appear in dissipated power originating from the excitations of hybridized substrate phonons.

We showed that if one graphene is pristine $\left(E_{F}=0\right)$ it causes the disappearance of the strong $2 \omega_{p}$ peak in the dissipated power. Moreover, for larger separations $a$ the doping causes shifts, appearance, and disappearance of many peaks originating from resonant coupling between hybridized electronic/phononic excitations in graphene/substrate slabs.

Obviously, when present, the Dirac plasmons are the dominant channels through which the energy between slabs can be transferred, so the studied model system strongly supports the possibility to control the energy or heat transfer between the slabs by tuning the graphene doping, e.g., by electrostatic gating. More radically, for zero doping $E_{F}=0$ the energy transfer can be locked and vice versa. In conclusion, it is expected that studies of energy transfer processes in the case of oscillating slabs, based on our complete theoretical description, will provide a supplementary and more practical approach as compared to those in parallel uniform motion.

\section{ACKNOWLEDGMENTS}

Two of the authors (V.D. and M.Š.) are grateful for the hospitality at the Donostia International Physics Center where this work was finalized and for useful discussions to J. Pendry, A. A. Lucas, S. Silkin, and I. Kupčić. V.D. acknowledges the support of the University of the Basque Country and the Spanish Ministerio de Ciencia y Tehnologia. V.D. also acknowledges the support of QuantiXLie Centre of Excellence, a project cofinanced by the Croatian Government and European Union through the European Regional Development Fund-the Competitiveness and Cohesion Operational Programme (Grant No. KK.01.1.1.01.0004).

\section{APPENDIX: GENERAL THEORY-UNIFORMLY MOVING SLABS}

\section{Van der Waals energy and force}

We shall first derive the van der Waals potential and force between two inequivalent slabs, described by their response

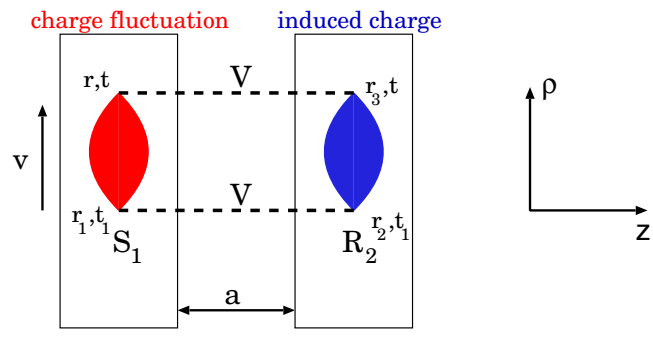

FIG. 11. Process in which charge density fluctuation is created in the left slab and induces a potential in the right slab.

functions $R_{1}$ and $R_{2}$, moving with relative parallel velocity $\mathbf{v}$ and separated by $a$, as can be seen in Fig. 11. In the following we shall briefly summarize the derivation presented in Ref. [26], modified to describe the most general case, i.e., for the slabs with different response functions $R_{1} \neq R_{2}$ and different temperatures $T_{1} \neq T_{2}$, including the case of graphene monolayers deposited on dielectric substrates. In the diagram in Fig. 11 the density fluctuation $S_{1}$ in the slab 1 couples to the induced charge described by $R_{2}$ inside the slab 2 , which results in the ground state energy shift:

$$
\begin{aligned}
E_{c}= & \int_{-\infty}^{\infty} d t_{1} \int d \mathbf{r} \int d \mathbf{r}_{1} \int d \mathbf{r}_{2} \int d \mathbf{r}_{3} S_{1}\left(\mathbf{r}, \mathbf{r}_{1}, t, t_{1}\right) \\
& \times V\left(\mathbf{r}, \mathbf{r}_{3}\right) R_{2}\left(\mathbf{r}_{3}, \mathbf{r}_{2}, t, t_{1}\right) V\left(\mathbf{r}_{2}, \mathbf{r}_{1}\right) .
\end{aligned}
$$

Here $V$ is the Coulomb potential, $S_{1}$ is the correlation function of the left slab, and $R_{2}$ is the response function of the right slab. We assume that the slab 1 is moving with velocity $\mathbf{v}$ so that the parallel coordinates in $S_{1}$ are transformed as

$$
\rho-\rho_{1} \rightarrow \rho-\rho_{1}-\mathbf{v}\left(t-t_{1}\right) .
$$

If we use translational invariance in time and in the parallel direction and perform the Fourier transform in parallel coordinates we find

$$
\begin{aligned}
E_{c}=\int_{-\infty}^{\infty} d t_{1} \int \frac{d \mathbf{Q}}{(2 \pi)^{2}} e^{-i \mathbf{Q} \mathbf{v}\left(t-t_{1}\right)} \\
\quad \times \int_{-\infty}^{\infty} d z d z_{1} d z_{2} d z_{3} S_{1}\left(\mathbf{Q}, z, z_{1}, t-t_{1}\right) V\left(\mathbf{Q}, z, z_{3}\right) \\
\quad \times R_{2}\left(\mathbf{Q}, z_{3}, z_{2}, t-t_{1}\right) V\left(\mathbf{Q}, z_{2}, z_{1}\right) .
\end{aligned}
$$

The Fourier transform in time gives:

$$
\begin{aligned}
E_{c}= & \int_{-\infty}^{\infty} \frac{d \omega}{2 \pi} \int \frac{d \mathbf{Q}}{(2 \pi)^{2}} \int_{-\infty}^{\infty} d z d z_{1} d z_{2} d z_{3} S_{1}\left(\mathbf{Q}, z, z_{1},|\omega|\right) \\
& \times V\left(\mathbf{Q}, z, z_{3}\right) R_{2}\left(\mathbf{Q}, z_{3}, z_{2}, \omega^{\prime}\right) V\left(\mathbf{Q}, z_{2}, z_{1}\right),
\end{aligned}
$$

where we have introduced $\omega^{\prime}=\omega+\mathbf{Q v}$. Because the charge densities in slabs 1 and 2 do not overlap, $z$ integrals in (A4) contribute only for $z_{3}>z$ and $z_{2}>z_{1}$, so that we can write

$$
\begin{aligned}
V\left(\mathbf{Q}, z, z_{3}\right) & =v_{Q} e^{-Q\left(z_{3}-z\right)}, \\
V\left(\mathbf{Q}, z_{2}, z_{1}\right) & =v_{Q} e^{-Q\left(z_{2}-z_{1}\right)},
\end{aligned}
$$

where $v_{Q}=\frac{2 \pi e^{2}}{Q}$. Also, because the polarizable crystal slabs are described by nonlocal response functions $R_{i}\left(\mathbf{Q}, \omega, z_{1}, z_{2}\right)$, which spread through $-\Delta<z_{1}, z_{2}<0$ and $i=1$ or 2 , the 
induced dynamically screened Coulomb potential at the point $z>0$ caused by the point charge at point $z^{\prime}>0$ is

$$
\begin{aligned}
& W_{i}^{\text {ind }}\left(\mathbf{Q}, \omega, z, z^{\prime}\right) \\
& \quad=v_{Q}^{2} e^{-Q\left(z+z^{\prime}\right)} \int_{-\infty}^{\infty} e^{Q\left(z_{1}+z_{2}\right)} R_{i}\left(\mathbf{Q}, \omega, z_{1}, z_{2}\right) d z_{1} d z_{2} .
\end{aligned}
$$

Surface excitations propagators are defined as $[50,51]$

$$
\begin{aligned}
D_{i}(Q, \omega) & =W_{i}^{i n d}\left(z=0, z^{\prime}=0\right) / v_{Q} \\
& =v_{Q} \int_{-\infty}^{\infty} e^{Q\left(z_{1}+z_{2}\right)} R_{i}\left(Q, \omega, z_{1}, z_{2}\right) d z_{1} d z_{2},
\end{aligned}
$$

i.e., they are proportional to the Coulomb potential at crystal surfaces $(z=0)$ driven by point charge placed at crystal surface $\left(z^{\prime}=0\right)$. Imaginary part of the surface excitation propagator (A6) contains information about intensities of all electronic excitations in the crystal, however, due to geometrical reasons the surface excitations are favorized. Therefore, after using (A6) and the definition of the surface correlation function

$$
S_{1}(\mathbf{Q},|\omega|)=v_{Q} \int_{-\infty}^{\infty} d z d z_{1} e^{Q z} S_{1}\left(\mathbf{Q},|\omega|, z, z_{1}\right) e^{Q z_{1}},
$$

expression (A4) can be written as

$$
E_{c}=\int_{-\infty}^{\infty} \frac{d \omega}{2 \pi} \int \frac{d \mathbf{Q}}{(2 \pi)^{2}} e^{-2 Q a} S_{1}(\mathbf{Q},|\omega|) \operatorname{Re} D_{2}\left(\mathbf{Q}, \omega^{\prime}\right),
$$

where we have used the fact that $\operatorname{Im} D_{2}(\mathbf{Q}, \omega)$ is an odd function of $\omega$ so that this term does not contribute. Moreover, after using the fluctuation-dissipation theorem which connects thermal/quantum mechanical charge density fluctuations and the dissipation, the surface correlation function $S$ (A7) can be connected with the imaginary part of the surface excitations propagator $\mathrm{D}(\mathrm{A} 6)$ as

$$
S_{1}(\mathbf{Q},|\omega|)=-\frac{\hbar}{\pi}\left[2 n_{1}(\omega)+1\right] \operatorname{Im} D_{1}(\mathbf{Q}, \omega),
$$

where $n_{1}(\omega)=1 /\left(e^{\beta_{1} \hbar \omega}-1\right)$ represents the Bose-Einstein distribution, $\beta=k_{B} T_{1}$, and $T_{1}$ is the temperature of slab 1 . After using (A9) the expression (A8) becomes

$$
\begin{aligned}
E_{c}= & -\frac{\hbar}{\pi} \int_{-\infty}^{\infty} \frac{d \omega}{2 \pi}\left[2 n_{1}(\omega)+1\right] \int \frac{d \mathbf{Q}}{(2 \pi)^{2}} e^{-2 Q a} \\
& \times \operatorname{Im} D_{1}(\mathbf{Q}, \omega) \operatorname{Re} D_{2}\left(\mathbf{Q}, \omega^{\prime}\right) .
\end{aligned}
$$

To this we have to add the contribution from the process in which the charge density fluctuation is created in the slab 2 . Because then slab 2 moves with parallel velocity $\mathbf{v}$ relative to slab 1 this contribution can be obtained from (A10) by exchanging $\mathbf{v} \rightarrow-\mathbf{v}$ and $1 \leftrightarrow 2$, and the result for the van der Waals energy is:

$$
\begin{aligned}
E_{c}= & -\frac{\hbar}{\pi} \int \frac{d \mathbf{Q}}{(2 \pi)^{2}} e^{-2 Q a} \int_{-\infty}^{\infty} \frac{d \omega}{2 \pi} \\
& \times\left\{\left[2 n_{1}(\omega)+1\right] \operatorname{Im} D_{1}(\mathbf{Q}, \omega) \operatorname{Re} D_{2}\left(\mathbf{Q}, \omega^{\prime}\right)\right. \\
& \left.+\left[2 n_{2}(\omega)+1\right] \operatorname{Im} D_{2}(\mathbf{Q}, \omega) \operatorname{Re} D_{1}\left(\mathbf{Q}, \omega^{\prime}\right)\right\} .
\end{aligned}
$$

In the lowest approximation we suppose that both layers are connected to heat baths of constant temperatures $T_{1}$ and $T_{2}$,

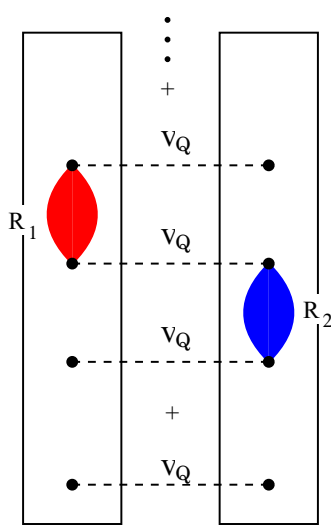

FIG. 12. Higher order processes.

so that in the thermodynamic equilibrium the physical values in both slabs narrowly fluctuate about their 'own' thermodynamic averages. These fluctuations couple and if $T_{1} \neq T_{2}$ it results in the heat stationary flow or diffusion. Our model does not include this stationary heat flow. Relative velocity causes unbalanced electrical field thermal and quantum mechanical fluctuations in separate slabs which causes 'work done' on the system and departure from the thermodynamic equilibrium. However, the system adiabatically adapts to those changes and thermalizes, so that it is never actually out of equilibrium. This 'work done' is equal to dissipated energy as will be discussed in Sec. A 2. $E_{c}$ given by (A11) includes only the lowest order processes shown in Fig. 11. If we want to include higher order processes shown in Fig. 12, we have to replace the interaction $v_{Q}$ which appears in $D_{1}$ :

$$
v_{Q} \rightarrow v_{Q}\left(1+D_{2} D_{1} e^{-2 Q a}+\ldots\right)=\frac{v_{Q}}{1-D_{2} D_{1} e^{-2 Q a}}
$$

and the one which appears in $D_{2}$ :

$$
v_{Q} \rightarrow v_{Q}\left(1+D_{1}^{*} D_{2}^{*} e^{-2 Q a}+\ldots\right)=\frac{v_{Q}}{1-D_{1}^{*} D_{2}^{*} e^{-2 Q a}}
$$

Considering that van der Waals energy represents the ground state energy shift relative to the ground state energies of two independent crystal slabs, in the final expression we have to integrate over the coupling constant $\lambda$ (the strength of interslab Coulomb interaction), as described in detail in Ref. [30], to find

$$
\begin{aligned}
E_{c}= & \hbar \int_{0}^{1} \frac{d \lambda}{\lambda} \int \frac{d \mathbf{Q}}{(2 \pi)^{2}} e^{-2 Q a} \int_{-\infty}^{\infty} \frac{d \omega}{2 \pi}\left[2 n_{1}(\omega)+1\right] \\
& \times\left[\frac{\lambda^{2} \operatorname{Im} D_{1}(\mathbf{Q}, \omega) \operatorname{Re} D_{2}\left(\mathbf{Q}, \omega^{\prime}\right)}{\left|1-\lambda^{2} e^{-2 Q a} D_{1}(\mathbf{Q}, \omega) D_{2}\left(\mathbf{Q}, \omega^{\prime}\right)\right|^{2}}+(1 \leftrightarrow 2)\right] .
\end{aligned}
$$


Notice that (A14) does not change for $\mathbf{v} \rightarrow-\mathbf{v}$. In order to do the $\lambda$ integration we transform this expression into:

$$
E_{c}=\hbar \int_{0}^{1} \frac{d \lambda}{\lambda} \int \frac{d \mathbf{Q}}{(2 \pi)^{2}} \int_{-\infty}^{\infty} \frac{d \omega}{2 \pi}\left[2 n_{1}(\omega)+1\right]\left\{\frac{\operatorname{Im} D_{1}(\mathbf{Q}, \omega) \operatorname{Re} D_{2}\left(\mathbf{Q}, \omega^{\prime}\right)}{\operatorname{Im}\left[D_{1}(\mathbf{Q}, \omega) D_{2}\left(\mathbf{Q}, \omega^{\prime}\right)\right]} \operatorname{Im} \frac{\lambda^{2} e^{-2 Q a} D_{1}(\mathbf{Q}, \omega) D_{2}\left(\mathbf{Q}, \omega^{\prime}\right)}{1-\lambda^{2} e^{-2 Q a} D_{1}(\mathbf{Q}, \omega) D_{2}\left(\mathbf{Q}, \omega^{\prime}\right)}+(1 \leftrightarrow 2)\right\}
$$

which finally gives the van der Waals energy in the case of unequal slabs and finite velocity:

$$
E_{c}(a)=\frac{\hbar}{2} \int \frac{d \mathbf{Q}}{(2 \pi)^{2}} \int_{-\infty}^{\infty} \frac{d \omega}{2 \pi} A\left(\mathbf{Q}, \omega, \omega^{\prime}\right)
$$

where

$$
A\left(\mathbf{Q}, \omega, \omega^{\prime}\right)=\left[2 n_{1}(\omega)+1\right] A_{12}\left(\mathbf{Q}, \omega, \omega^{\prime}\right)+(1 \leftrightarrow 2)
$$

and

$$
\begin{aligned}
A_{i j}\left(\mathbf{Q}, \omega, \omega^{\prime}\right)= & \frac{\operatorname{Im} D_{i}(\mathbf{Q}, \omega) \operatorname{Re} D_{j}\left(\mathbf{Q}, \omega^{\prime}\right)}{\operatorname{Im}\left[D_{i}(\mathbf{Q}, \omega) D_{j}\left(\mathbf{Q}, \omega^{\prime}\right)\right]} \\
& \times \operatorname{Im} \ln \left[1-e^{-2 Q a} D_{i}(\mathbf{Q}, \omega) D_{j}\left(\mathbf{Q}, \omega^{\prime}\right)\right]
\end{aligned}
$$

One can easily derive the limiting cases. For $T_{1}=T_{2}=$ $02 n_{1}(\omega)+1 \rightarrow \operatorname{sgn} \omega$. For $\mathbf{v}=0$ and $\omega^{\prime}=\omega$ the expression (A17) becomes (for $T=0$ ):

$$
A\left(\mathbf{Q}, \omega=\omega^{\prime}\right)=\operatorname{Im} \ln \left[1-e^{-2 Q a} D_{1}(\mathbf{Q}, \omega) D_{2}(\mathbf{Q}, \omega)\right] .
$$

For $\mathbf{v} \neq 0$ but $D_{1}=D_{2}=D$ it becomes:

$$
\begin{aligned}
A\left(\mathbf{Q}, \omega, \omega^{\prime}\right)= & 2 \frac{\operatorname{Im} D(\mathbf{Q}, \omega) \operatorname{Re} D\left(\mathbf{Q}, \omega^{\prime}\right)}{\operatorname{Im}\left[D(\mathbf{Q}, \omega) D\left(\mathbf{Q}, \omega^{\prime}\right)\right]} \\
& \times \operatorname{Im} \ln \left[1-e^{-2 Q a} D(\mathbf{Q}, \omega) D\left(\mathbf{Q}, \omega^{\prime}\right)\right] .
\end{aligned}
$$

From the van der Waals potential $E_{c}(a)$ we can derive the perpendicular attractive force $F_{\perp}(a)$ between two moving slabs:

$$
\begin{aligned}
F_{\perp}(a) & =-\frac{d E_{c}(a)}{d a} \\
& =\hbar \int \frac{d \mathbf{Q}}{(2 \pi)^{2}} Q e^{-2 Q a} \int_{-\infty}^{\infty} \frac{d \omega}{2 \pi} B\left(\mathbf{Q}, \omega, \omega^{\prime}\right),
\end{aligned}
$$

where

$$
B\left(\mathbf{Q}, \omega, \omega^{\prime}\right)=\left[2 n_{1}(\omega)+1\right] B_{12}\left(\mathbf{Q}, \omega, \omega^{\prime}\right)+(1 \rightarrow 2)
$$

and

$$
B_{i j}\left(\mathbf{Q}, \omega, \omega^{\prime}\right)=\frac{\operatorname{Im} D_{i}(\mathbf{Q}, \omega) \operatorname{Re} D_{j}\left(\mathbf{Q}, \omega^{\prime}\right)}{\left|1-e^{-2 Q a} D_{i}(\mathbf{Q}, \omega) D_{j}\left(\mathbf{Q}, \omega^{\prime}\right)\right|^{2}} .
$$

Again for $\mathbf{v}=0$ and $T_{1}=T_{2}=0$ the expression (A22) becomes

$$
B\left(\mathbf{Q}, \omega=\omega^{\prime}\right)=\frac{\operatorname{Im}\left[D_{1}(\mathbf{Q}, \omega) D_{2}(\mathbf{Q}, \omega)\right]}{\left|1-e^{-2 Q a} D_{1}(\mathbf{Q}, \omega) D_{2}(\mathbf{Q}, \omega)\right|^{2}},
$$

and for $\mathbf{v} \neq 0$ but $D_{1}=D_{2}=D$ it becomes

$$
B\left(\mathbf{Q}, \omega, \omega^{\prime}\right)=2 \frac{\operatorname{Im} D(\mathbf{Q}, \omega) \operatorname{Re} D\left(\mathbf{Q}, \omega^{\prime}\right)}{\left|1-e^{-2 Q a} D(\mathbf{Q}, \omega) D\left(\mathbf{Q}, \omega^{\prime}\right)\right|^{2}} .
$$

We note that for $\mathbf{v}=0$ our results agree with the previous ones, but for $\mathbf{v} \neq 0$ they differ from those in Refs. [1,14]. The functions $A$ and $B$ will also appear in the same form in the expressions for van der Waals potential and force between the oscillating slabs, but with the different choice for $\omega^{\prime}$.

We can verify, using spectral representations for $R e D$ 's, that our results correspond exactly to the well known result for the van der Waals attraction between two moving or oscillating objects in the lowest order [29], e.g., for $T=0$ :

$$
\begin{aligned}
E_{c}^{(2)}= & \frac{\hbar}{2} \int \frac{d \mathbf{Q}}{(2 \pi)^{2}} e^{-2 Q a} \int_{-\infty}^{\infty} \frac{d \omega}{2 \pi} \operatorname{sgn} \omega \\
& \times\left[\operatorname{Im} D_{1}(\mathbf{Q}, \omega) \operatorname{Re} D_{2}\left(\mathbf{Q}, \omega^{\prime}\right)\right. \\
& \left.+\operatorname{Im} D_{2}(\mathbf{Q}, \omega) \operatorname{Re} D_{1}\left(\mathbf{Q}, \omega^{\prime}\right)\right],
\end{aligned}
$$

where $\omega^{\prime}=\omega+\Delta \omega$ and $\Delta \omega=\mathbf{Q v}$ for uniform motion or $\Delta \omega=n \omega_{0}$ for an oscillator. The frequency integral can be rewritten as:

$$
\begin{aligned}
\int_{-\infty}^{\infty} & \frac{d \omega}{2 \pi} \operatorname{sgn} \omega\left[\operatorname{Im} D_{1}(\mathbf{Q}, \omega) \operatorname{Re} D_{2}\left(\mathbf{Q}, \omega^{\prime}\right)\right. \\
& \left.+\operatorname{Im} D_{2}(\mathbf{Q}, \omega) \operatorname{Re} D_{1}\left(\mathbf{Q}, \omega^{\prime}\right)\right] \\
= & 4 \int_{0}^{\infty} d \omega d \nu \frac{\operatorname{Im} D_{1}(\mathbf{Q}, \omega) \operatorname{Im} D_{2}(\mathbf{Q}, v)}{\omega+v+\Delta \omega},
\end{aligned}
$$

which is exactly the lowest order term in (A16).

\section{Dissipated power and friction force}

Now we shall calculate the energy dissipated by the two slabs in parallel uniform motion following the derivation in Ref. [26]. Suppose that the left slab is moving parallel to the right one with relative velocity $\mathbf{v}$ and that a charge density fluctuation is spontaneously created in the left slab at the moment $t_{1}$ (Fig. 1). Propagating in time between $t_{1}$ and $t$ it induces charge density fluctuations in the right slab with which it can subsequently interact. In such a process the left slab can be considered as a field source which heats itself, and in analogy with Eqs. (3) and (4) of Ref. [26], the energy loss rate operator in this process can be written as

$$
\begin{aligned}
\hat{P}_{12} & =\int d \mathbf{r} \int d \mathbf{r}_{1} \int_{-\infty}^{\infty} d t_{1} \hat{\rho}(\mathbf{r}, t) V\left(\mathbf{r}, \mathbf{r}_{3}\right) \\
& \otimes \frac{d}{d t} D_{2}\left(\mathbf{r}_{3}, \mathbf{r}_{2}, t, t_{1}\right) \otimes V\left(\mathbf{r}_{2}, \mathbf{r}_{1}\right) \hat{\rho}\left(\mathbf{r}_{1}, t_{1}\right)
\end{aligned}
$$

where $D_{2}$ is the retarded response function of the right slab and $\hat{\rho}(\mathbf{r}, t)$ and $\hat{\rho}(\mathbf{r}, t)$ are density operators which represent quantum mechanical charge density fluctuations created and annihilated at points $\left(\mathbf{r}_{1}, t_{1}\right)$ and $(\mathbf{r}, t)$, respectively. Energy absorption rate in the left slab (driven by the field fluctuations produced in the same slab but reflected from the right slab) can be obtained by taking the ground state matrix element of 


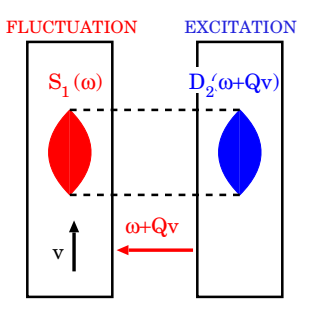

(a)

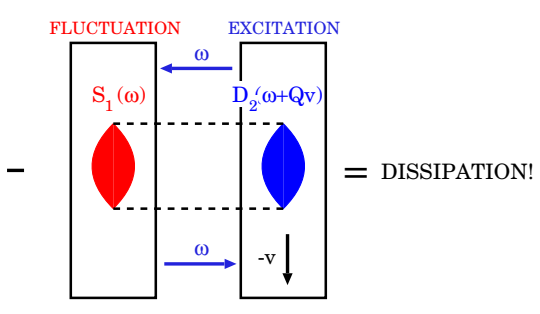

(b)
FIG. 13. Process in which the energy $\omega+\mathbf{v Q}$ is absorbed in the left slab (a), and the process in which the energy $\omega$ fluctuates between left and right slabs (b).

Eq. (A28)

$$
\begin{aligned}
P_{12} & =\left\langle\hat{P}_{12}(t)\right\rangle=\int d \mathbf{r} \int d \mathbf{r}_{1} \int_{-\infty}^{\infty} d t_{1} S_{1}\left(\mathbf{r}, \mathbf{r}_{1}, t, t_{1}\right) V\left(\mathbf{r}, \mathbf{r}_{3}\right) \\
& \otimes \frac{d}{d t} D_{2}\left(\mathbf{r}_{3}, \mathbf{r}_{2}, t, t_{1}\right) \otimes V\left(\mathbf{r}_{2}, \mathbf{r}_{1}\right),
\end{aligned}
$$

where

$$
S_{1}\left(\mathbf{r}, \mathbf{r}_{1}, t, t_{1}\right)=\left\langle\hat{\rho}(\mathbf{r}, t) \hat{\rho}\left(\mathbf{r}_{1}, t_{1}\right)\right\rangle+\left\langle\hat{\rho}\left(\mathbf{r}_{1}, t_{1}\right) \hat{\rho}(\mathbf{r}, t)\right\rangle
$$

is the correlation function of the left slab which represents real charge density fluctuation. Equation (A29) can be illustrated by the the Feynman diagram in Fig. 13. We note that in the inertial system of the right slab the charge density in the left slab, apart from the fluctuations, has an additional parallel component of motion, so all parallel coordinates in the left slab have to be transformed as in (A2). Explicitly, the correlation function (A30) becomes

$$
S_{1}\left(\mathbf{r}, \mathbf{r}_{1}, t, t_{1}\right)=S_{1}\left(z, z_{1}, \boldsymbol{\rho}-\mathbf{v} t, \rho_{1}-\mathbf{v} t_{1}, t, t_{1}\right) .
$$

After inserting (A31) into (A29) and the Fourier transformation in parallel coordinates and in time we get the formula for energy absorption rate in the left slab per unit surface area

$$
\begin{aligned}
P_{12}= & -i \hbar \int_{-\infty}^{\infty} d z \int_{-\infty}^{\infty} d z_{1} \int \frac{d \mathbf{Q}}{(2 \pi)^{2}} \int_{-\infty}^{\infty} \frac{d \omega}{2 \pi} \\
& \times \omega^{\prime} S_{1}\left(\mathbf{Q},|\omega|, z, z_{1}\right) V\left(\mathbf{Q}, z, z_{3}\right) \\
\otimes & D_{2}\left(\mathbf{Q}, \omega^{\prime}, z_{3}, z_{2}\right) \otimes V\left(\mathbf{Q}, z_{2}, z_{1}\right) .
\end{aligned}
$$

After using the definitions (A5), (A6), and (A7) equation (A32) can be written as

$$
P_{12}=-i \hbar \int \frac{d \mathbf{Q}}{(2 \pi)^{2}} \int_{-\infty}^{\infty} \frac{d \omega}{2 \pi} e^{-2 Q a} S_{1}(\mathbf{Q},|\omega|) \omega^{\prime} D_{2}\left(\mathbf{Q}, \omega^{\prime}\right) .
$$

Using the connection (A9) between the surface correlation function $S$ and the imaginary part of the surface excitation propagator $D$, equation (A33) can be written as

$$
\begin{aligned}
P_{12}= & -i \hbar \int \frac{d \mathbf{Q}}{(2 \pi)^{2}} \int_{-\infty}^{\infty} \frac{d \omega}{2 \pi} e^{-2 Q a} \omega^{\prime}\left[2 n_{1}(\omega)+1\right] \\
& \times \operatorname{Im} D_{1}(\mathbf{Q}, \omega) D_{2}\left(\mathbf{Q}, \omega^{\prime}\right) .
\end{aligned}
$$

Finally, as the imaginary part of surface excitation propagator (A9) is an odd function of frequency, $P_{12}$ given by Eq. (A34) is a real quantity

$$
\begin{aligned}
P_{12}= & \hbar \int \frac{d \mathbf{Q}}{(2 \pi)^{2}} e^{-2 Q a} \int_{-\infty}^{\infty} \frac{d \omega}{2 \pi} \omega^{\prime}\left[2 n_{1}(\omega)+1\right] \\
& \times \operatorname{Im} D_{1}(\mathbf{Q}, \omega) \operatorname{Im} D_{2}\left(\mathbf{Q}, \omega^{\prime}\right) .
\end{aligned}
$$

The Feynman diagram which illustrates equation (A35) is shown in Fig. 13(a).

We see that if the charge fluctuation is created with the energy $\omega$ it can create excitations in the right slab with the energy $\omega^{\prime}=\omega+\mathbf{v Q}$. This is expected, namely, $\omega$ is the energy in the inertial system of the left slab, but in the inertial system of the right slab it is Doppler shifted by $\mathbf{v Q}$.

In (A35) we have calculated the energy absorbed in the left slab. However, the part of this energy represents the quantum mechanical fluctuations, i.e., the energy which just fluctuates between two slabs. We can calculate this part of the energy which fluctuates between the slabs by going to the inertial system of the left slab and forgetting for the moment the right one. Sitting in the inertial system of the left slab we know that it is in the quantum-mechanical (and thermodynamical) equilibrium with the environment (in this case with the right slab). So, the energy just fluctuates between the left slab and the environment, i.e., the energy which is given to the environment is exactly equal to the energy which is received from the environment. This energy which comes from the environment and is 'absorbed' in the left slab can be calculated using exactly the same ideas as before, except that now the right slab is moving with the velocity $-\mathbf{v}$ and the left one is at rest. Therefore, following the same procedure (A28)-(A35) with the response functions of the right slab transformed as

$$
D_{2}\left(\mathbf{r}, \mathbf{r}_{1}, t, t_{1}\right)=D_{2}\left(z, z_{1}, \boldsymbol{\rho}+\mathbf{v} t, \boldsymbol{\rho}_{1}+\mathbf{v} t_{1}, t, t_{1}\right),
$$

we obtain the energy that fluctuates between two slabs

$$
\begin{aligned}
P_{12}^{\prime}= & \hbar \int \frac{d \mathbf{Q}}{(2 \pi)^{2}} e^{-2 Q a} \int_{-\infty}^{\infty} \frac{d \omega}{2 \pi} \omega\left[2 n_{1}(\omega)+1\right] \\
& \times \operatorname{Im} D_{1}(\mathbf{Q}, \omega) \operatorname{Im}_{2}\left(\mathbf{Q}, \omega^{\prime}\right) .
\end{aligned}
$$

The dissipated (heating) power can be calculated by extracting from $P_{12}$ this fluctuating component $P_{12}^{\prime}$

$$
\begin{aligned}
P_{1}= & P_{12}-P_{12}^{\prime}=\hbar \mathbf{v} \int \frac{d \mathbf{Q}}{(2 \pi)^{2}} \mathbf{Q} e^{-2 Q a} \\
& \times \int_{-\infty}^{\infty} \frac{d \omega}{2 \pi}\left[2 n_{1}(\omega)+1\right] \operatorname{Im} D_{1}(\mathbf{Q}, \omega) \operatorname{Im} D_{2}\left(\mathbf{Q}, \omega^{\prime}\right) .
\end{aligned}
$$

Expression (A38) represents the dissipated power if the charge fluctuation is spontaneously created in the left slab. However, the charge fluctuation can also be spontaneously created in the right slab, then the corresponding dissipated power can be obtained from (A38) with $1 \leftrightarrow 2$ and $\mathbf{v} \leftrightarrow-\mathbf{v}$. Therefore the total dissipated power can be written as

$$
\begin{aligned}
P= & P_{1}+P_{2}=\hbar \mathbf{v} \int \frac{d \mathbf{Q}}{(2 \pi)^{2}} \mathbf{Q} e^{-2 Q a} \int_{-\infty}^{\infty} \frac{d \omega}{2 \pi} \operatorname{sgn}(\omega) \\
& \times\left\{\left[2 n_{1}(\omega)+1\right] \operatorname{Im} D_{1}(\mathbf{Q}, \omega) \operatorname{Im} D_{2}\left(\mathbf{Q}, \omega^{\prime}\right)\right. \\
& \left.+\left[2 n_{2}(\omega)+1\right] \operatorname{Im} D_{1}\left(\mathbf{Q}, \omega^{\prime}\right) \operatorname{Im} D_{2}(\mathbf{Q}, \omega)\right\} .
\end{aligned}
$$


This result can be transformed by changing the arguments $\omega+$ $\mathbf{v Q} \rightarrow \omega$ and $\mathbf{Q} \rightarrow-\mathbf{Q}$ in the second term of (A39) and the frequency integration becomes

$$
\begin{aligned}
& 2 \hbar \mathbf{v} \mathbf{Q} \int_{-\infty}^{\infty} \frac{d \omega}{2 \pi}\left[n_{1}(\omega)-n_{2}(\omega+\mathbf{v} \mathbf{Q})\right] \\
& \quad \times \operatorname{Im} D_{1}(\mathbf{Q}, \omega) \operatorname{Im} D_{2}(\mathbf{Q}, \omega+\mathbf{v} \mathbf{Q}) .
\end{aligned}
$$

For $T=0$ this reduces to the well known result [26]

$$
2 \hbar \mathbf{v} \mathbf{Q} \int_{0}^{\mathbf{Q v}} \frac{d \omega}{2 \pi} \operatorname{Im} D_{1}(\mathbf{Q}, \omega) \operatorname{Im} D_{2}(\mathbf{Q}, \mathbf{v} \mathbf{Q}-\omega)
$$

As in the case of van der Waals energy in Sec. A 1 the higher order terms can be included by replacing $v_{Q}$ 's in $D_{i}$ 's in (A40) by an infinite series [(A12),(A13)], as also shown in Fig. 12, so that we get

$$
P=2 \hbar \int \frac{d \mathbf{Q}}{(2 \pi)^{2}} e^{-2 Q a} \mathbf{v} \mathbf{Q} \int_{-\infty}^{\infty} \frac{d \omega}{2 \pi} C\left(\mathbf{Q}, \omega, \omega^{\prime}\right),
$$

where

$$
\begin{aligned}
C\left(\mathbf{Q}, \omega, \omega^{\prime}\right)= & {\left[n_{1}(\omega)-n_{2}\left(\omega^{\prime}\right)\right] } \\
& \times \frac{\operatorname{Im} D_{1}(\mathbf{Q}, \omega) \operatorname{Im} D_{2}\left(\mathbf{Q}, \omega^{\prime}\right)}{\left|1-e^{-2 Q a} D_{1}(\mathbf{Q}, \omega) D_{2}\left(\mathbf{Q}, \omega^{\prime}\right)\right|^{2}}
\end{aligned}
$$

and $\omega^{\prime}=\mathbf{v Q}-\omega$. Dissipated power can be simply related to the friction force $\mathbf{F}$ by $P=-\mathbf{F v}$, so that

$$
\mathbf{F}=-\hbar \int \frac{d \mathbf{Q}}{(2 \pi)^{2}} e^{-2 Q a} \mathbf{Q} \int_{-\infty}^{\infty} \frac{d \omega}{2 \pi} C\left(\mathbf{Q}, \omega, \omega^{\prime}\right) .
$$

Obviously, for $\mathbf{v} \rightarrow 0$ both $P$ and $\mathbf{F}$ vanish. This result agrees with Pendry's alternative derivation [1,17].

The above derivation repeats and generalizes some previously well known results [1,14-18,26]. We should note that this derivation takes into account not the local but the full microscopically calculated nonlocal response functions $R_{i}\left(\mathbf{Q}, \omega, z, z^{\prime}\right) ; i=1,2$. However, its main purpose is to facilitate the derivation of analogous results for the oscillating slabs in Sec. II.
[1] J. B. Pendry, J. Phys.: Condens. Matter 9, 10301 (1997).

[2] A. I. Volokitin and B. N. J. Persson, Phys. Rev. Lett. 106, 094502 (2011).

[3] A. I. Volokitin, Phys. Rev. B 94, 235450 (2016).

[4] A. I. Volokitin and B. N. J. Persson, JETP Lett. 98, 143 (2013).

[5] A. I. Volokitin and B. N. J. Persson, Phys. Rev. B 78, 155437 (2008); 81, 239901(E) (2010).

[6] Z. Chao-Jie, Z. Ting, D. Xin-Peng, W. Tong-Biao, and L. Nian-Hua, Acta Phys. Sin. 65, 236801 (2016).

[7] Tong-Biao Wang, Nian-Hua Liu, Jiang-Tao, and LiuTian-Bao Yu, Eur. Phys. J. B 87, 185 (2014).

[8] M. Bordag, I. Fialkovskiy, and D. Vassilevich, Phys. Rev. B 93, 075414 (2016).

[9] S. Kuehn, R. F. Loring, and J. A. Marohn, Phys. Rev. Lett. 96, 156103 (2006).

[10] B. C. Stipe, H. J. Mamin, T. D. Stowe, T. W. Kenny, and D. Rugar, Phys. Rev. Lett. 87, 096801 (2001).

[11] K. Saitoh, K. Hayashi, Y. Shibayama, and K. Shirahama, Phys. Rev. Lett. 105, 236103 (2010).

[12] G. Zolfagharkhani, A. Gaidarzhy, Seung-Bo Shim, R. L. Badzey, and P. Mohanty, Phys. Rev. B 72, 224101 (2005).

[13] For an interesting review of time dependent phenomena at surfaces, such as charge density fluctuations induced by suddenly appeared point charge, see R. Diez Muino, D. Sanchez-Portal, V. M. Silkin, E. V. Chulkov, and P. M. Echenique, PNAS 108, 971 (2011).

[14] B. N. J. Persson and Z. Zhang, Phys. Rev. B 57, 7327 (1998).

[15] A. I. Volokitin and B. N. J. Persson, Rev. Mod. Phys. 79, 1291 (2007).

[16] A. I. Volokitin and B. N. J. Persson, J. Phys.: Conf. Ser. 291, 012018 (2011).

[17] J. B. Pendry, New J. Phys. 12, 033028 (2010).

[18] T. G. Philbin and U. Leonhardt, New J. Phys. 11, 033035 (2009).
[19] G. L. Klimchitskaya, U. Mohideen, and V. M. Mostepanenko, Rev. Mod. Phys. 81, 1827 (2009).

[20] Bo E. Sernelius, Europhys. Lett. 95, 57003 (2011); Phys. Rev. B 85, 195427 (2012).

[21] J. Sarabadani, A. Naji, R. Asgari, and R. Podgornik, Phys. Rev. B 84, 155407 (2011).

[22] M. Bordag, G. L. Klimchitskaya, and V. M. Mostepanenko, Phys. Rev. B 86, 165429 (2012).

[23] I. V. Fialkovsky, V. N. Marachevsky, and D. V. Vassilevich, Phys. Rev. B 84, 035446 (2011).

[24] I. Kupčić, Phys. Rev. B 90, 205426 (2014).

[25] I. Kupčić, Phys. Rev. B 91, 205428 (2015).

[26] V. Despoja, P. M. Echenique, M. Šunjić, Phys. Rev. B 83, 205424 (2011).

[27] Brief preliminary report (V. Despoja, Pedro M. Echenique, and M. Šunjić: Some quantum mechanical aspects of frictional force between parallel metallic slabs) was presented at the conference Passion for knowwledge-Passion for electrons (Donostia-San Sebastian, 29 September-1 October 2010), with the application to metallic slabs treated in the surface plasmon model.

[28] Monography, by A. I. Volokitin and B. N. J. Persson, Electromagnetic fluctuations at the nanoscale. Theory and applications (Springer-Verlag, Berlin, Heidelberg, 2017).

[29] J. F. Annett and P. M. Echenique, Phys. Rev. B 34, 6853 (1986).

[30] V. Despoja, M. Šunjić, and L. Marušić, Phys. Rev. B 75, 045422 (2007).

[31] T. Marinković, I. Radović, D. Borka, and Z. L. Mišković, Phys. Lett. A 379, 377 (2015).

[32] T. Marinković, I. Radović, D. Borka, and Z. L. Mišković, Plasmonics 10, 1741 (2015).

[33] V. Despoja, T. Djordjević, L. Karbunar, I. Radović, and Z. L. Mišković, Phys. Rev. B 96, 075433 (2017).

[34] D. Novko, M. Šunjić, and V. Despoja, Phys. Rev. B 93, 125413 (2016). 
[35] The dielectric substrate screened interaction $\tilde{v}_{Q}$ can be obtained by solving Laplace equation $\left\{\frac{\partial^{2}}{\partial z^{2}}-Q^{2}\right\} \phi_{z^{\prime}}(z)=4 \pi \delta\left(z-z^{\prime}\right)$ and applying boundary conditions $D_{n}^{i}=D_{n}^{j}$ and $\epsilon_{i} E_{t}^{i}=\epsilon_{j} E_{t}^{j}$, where $i \neq j= \pm \Delta / 2 \pm 0^{+}, \epsilon_{ \pm \Delta / 2 \mp 0^{+}}=\epsilon(\omega)$ and $\epsilon_{ \pm \Delta / 2 \pm 0^{+}}=$ 1. Finally, $\tilde{v}_{Q}=\phi_{ \pm \Delta / 2}( \pm \Delta / 2)$.

[36] B. Wunsch, T. Stauber, F. Sols, and F. Guinea, New J. Phys. 8, 318 (2006).

[37] E. H. Hwang, and S. Das Sarma, Phys. Rev. B 75, 205418 (2007); 80, 205405 (2009).

[38] V. Despoja, D. J. Mowbray, D. Vlahović, and L. Marušić, Phys. Rev. B 86, 195429 (2012).

[39] V. Despoja, D. Novko, K. Dekanić, M. Šunjić, and L. Marušić, Phys. Rev. B 87, 075447 (2013).

[40] Combining the continuity equation $-i \omega \rho=\nabla \mathbf{j}$, the formula for induced current in the local limit $\mathbf{j}=\sigma(\omega) \mathbf{E}^{T O T}$, the $\mathbf{E}^{T O T}=$ $-\nabla \phi^{T O T}$, Fourier transformation in $\mathbf{Q}$ space, and using the definition $\rho(Q, \omega)=\chi^{0}(Q, \omega) \phi^{T O T}(Q, \omega)$, one can obtain the relation $\chi^{0}(Q, \omega)=\frac{Q^{2}}{i \omega} \sigma(\omega)$.

[41] L. A. Falkovsky and S. S. Pershoguba, Phys. Rev. B 76, 153410 (2007).
[42] R. Saito, G. Dresselhaus, and M. S. Dresselhaus, Physical Properties of Carbon Nanotubes (Imperial College Press, London, 1998).

[43] P. Giannozzi, S. Baroni, N. Bonini, M. Calandra, R. Car, C. Cavazzoni, D. Ceresoli, G. L. Chiarotti, M. Cococcioni, I. Dabo et al., J. Phys.: Condens. Matter 21, 395502 (2009).

[44] N. Troullier and J. L. Martins, Phys. Rev. B 43, 1993 (1991).

[45] J. P. Perdew and A. Zunger, Phys. Rev. B 23, 5048 (1981).

[46] H. J. Monkhorst and J. D. Pack, Phys. Rev. B 13, 5188 (1976).

[47] M. V. Fischetti, D. A. Neumayer, and E. A. Cartier, J. Appl. Phys. 90, 4587 (2001).

[48] M. Ishigami, J. H. Chen, W. G. Cullen, M. S. Fuhrer, and E. D. Williams, Nano Lett. 7, 1643 (2007).

[49] V. Despoja, V. M. Silkin, P. M. Echenique, and M. Šunjić, Phys. Rev. B 92, 125424 (2015).

[50] L. Marušić and M. Šunjić, Phys. Scr. 63, 336 (2001).

[51] L. Marušić, V. Despoja, and M. Šunjić, J. Phys.: Condens. Matter 18, 4253 (2006). 